\title{
Defect-based local error estimators for high-order splitting methods involving three linear operators
}

\author{
Winfried Auzinger • Othmar Koch . \\ Mechthild Thalhammer
}

The final publication is available at Springer via http://dx. doi.org/10.1007/s11075-015-0032-4.

\begin{abstract}
Prior work on high-order exponential operator splitting methods is extended to evolution equations defined by three linear operators. A posteriori local error estimators are constructed via a suitable integral representation of the local error involving the defect associated with the splitting solution and quadrature approximation via Hermite interpolation. In order to prove asymptotical correctness, a multiple integral representation involving iterated defects is deduced by repeated application of the variation-of-constant formula. The error analysis within the framework of abstract evolution equations provides the basis for concrete applications. Numerical examples for initial-boundary value problems of Schrödinger and of parabolic type confirm the asymptotical correctness of the proposed a posteriori error estimators.
\end{abstract}

Keywords Linear evolution equations - Time integration methods · High-order exponential operator splitting methods · Local error · A posteriori local error estimators

Mathematics Subject Classification (2000) 65J10, 65L05, 65M12, 65M15

\section{Introduction}

Evolution equations with the right-hand side composed of three vector fields naturally suggest splitting into three operators. Relevant examples are for instance given by reaction-diffusion-advection equations. Applications fields where this type of problems appears are, for instance, chemical reactor theory [1], fishery [2], or population dynamics [3]. Furthermore, dimensional splitting into three operators may promise computational advantages in the numerical solution of nonlinear Schrödinger equations.

Winfried Auzinger . Othmar Koch

Institute for Analysis and Scientific Computing, Vienna University of Technology

Wiedner Hauptstraße 8-10, A-1040 Wien, Austria

E-mail: w.auzinger@tuwien.ac.at, othmar@othmar-koch.org

Mechthild Thalhammer

Institut für Mathematik, Leopold-Franzens Universität Innsbruck

Technikerstraße 13/VII, A-6020 Innsbruck, Austria

E-mail: mechthild.thalhammer@uibk.ac.at 
For a recent overview on related numerical methods for (rotating) multi-component Bose-Einstein condensates we refer to [8], see also earlier work [4-7].

The generalization of the simplest splitting schemes (first-order Lie-Trotter splitting, second-order Strang splitting) to more than two operators is straightforward. Higher-order schemes can be constructed via composition. The construction of optimized higher-order schemes requires the solution of a nonlinear system of order conditions as for instance described in [9].

Given a splitting scheme involving three operators, our objective is to construct a practical local error estimator and to prove its asymptotical correctness. To this end the defect-based approach developed in [9,11-13] is extended to the case of three operators. This idea is rather universally applicable to one-step schemes, and it is of particular interest in the present context because other ways of estimating the error, for example using pairs of embedded formulas as proposed in [10], are difficult to realize and tend to be inefficient whenever three sub-operators are involved and higher-order schemes, e.g. of order $p=4$, are considered.

The motivation for our investigations is attributed to the fact that robust strategies for step-size adaptation according to the local error estimators are vital for the efficient time integration of evolution equations. To this end, reliable estimation and control of the local splitting error is necessary. The practical issues of adaptive time-stepping will not be addressed here, however.

To create a framework for this nontrivial extension of our prior approach [9,11-13], we consider an abstract linear evolution equation where the operator on the right-hand side is split into three parts,

$$
\left\{\begin{array}{l}
u^{\prime}(t)=H u(t)=A u(t)+B u(t)+C u(t), \quad t \geq 0 \\
\mathrm{u}(0) \text { given, }
\end{array}\right.
$$

and where the exact flow

$$
\mathcal{E}_{H}(t) u=\mathrm{e}^{t H} u
$$

is approximated, over a time increment $t>0$, by an $s$-stage exponential splitting method of the form

$$
\mathcal{S}(t) u=\mathcal{S}_{s}(t) \mathcal{S}_{s-1}(t) \cdots \mathcal{S}_{1}(t) u,
$$

with subflows

$$
\mathcal{S}_{j}(t)=\mathcal{E}_{C_{j}}(t) \mathcal{E}_{B_{j}}(t) \mathcal{E}_{A_{j}}(t)=\mathrm{e}^{t C_{j}} \mathrm{e}^{t B_{j}} \mathrm{e}^{t A_{j}}
$$

Here, we denote

$$
A_{j}=a_{j} A, \quad B_{j}=b_{j} B, \quad C_{j}=c_{j} C,
$$

with appropriate real or complex coefficients $\left(a_{j}, b_{j}, c_{j}\right)_{j=1}^{s}$.

As a first step, we deduce an exact representation of the local error operator

$$
\mathcal{L}(t)=\mathcal{S}(t)-\mathcal{E}_{H}(t),
$$

which subsequently serves as the basis for the construction and analysis of a posteriori local error estimators in particular application cases. For this purpose we extend our investigations from [13] (particularly Section 6) where the case of splitting into two suboperators was considered. In [13] the rich combinatorial structure of the local error was studied, and estimates for the local error have been obtained in a recursive way. For the case of three operators, an even more complex combinatorial structure emerges which we describe in detail. As this structure cannot be reduced to the case of two 
operators, an extended framework for the analysis needs to be created. We provide a complete analysis for linear problems; the general ideas related to the local error structure and a posteriori error estimation are the same in the nonlinear case, but technicalities abound, see for instance the discussion of splitting into two operators in [11].

Within our abstract setting we are not specific about the underlying function spaces. We tacitly assume that all occurring operations are well-defined. The notation $\mathscr{O}(\cdot)$ is to be understood in the sense that the stated order holds assuming boundedness of the quantities appearing in the error constants.

The paper is organized as follows: In Section 2 we describe the general setting and give some remarks on order conditions. In Section 3 we list examples of lower order schemes and their properties. Section 4 is devoted to a representation of the local error for the first-order Lie-Trotter splitting method and serves to indicate the general approach. In Section 5 we give a detailed analysis of the recursive combinatorial structure of the local error for general higher-order multi-composition methods. A related a posteriori error estimator is studied in Section 6; in particular it is demonstrated that this estimator is asymptotically correct under natural regularity requirements. Comments on the practical realization and the extension to nonlinear evolution equations are included in Section 7. Numerical examples for initial-boundary value problems of Schrödinger and of parabolic type confirming asymptotical correctness of the a posteriori local error estimators are finally given in Section 8.

\section{Problem setting and notation; the order of a splitting method}

\subsection{Notation and preliminaries}

For the sake of brevity we often omit the argument $t$ if the context allows. We write

$$
\mathcal{S}_{j}=\mathcal{S}_{j}^{[0]}, \quad \mathcal{S}=\mathcal{S}^{[0]} .
$$

Let

$$
\mathcal{V}_{j}=\mathcal{V}_{j}^{[0]}:=\mathcal{E}_{A_{j}}, \quad \mathcal{W}_{j}=\mathcal{W}_{j}^{[0]}:=\mathcal{E}_{C_{j}} \mathcal{E}_{B_{j}}
$$

such that $\mathcal{S}_{j}^{[0]}=\mathcal{W}_{j}^{[0]} \mathcal{V}_{j}^{[0]}$.

Introducing the defect operator

$$
\delta(\mathcal{X})=\frac{\mathrm{d}}{\mathrm{d} t} \mathcal{X}-H \mathcal{X}
$$

the exact evolution operator $\mathcal{E}_{H}(t)$ satisfies $\delta\left(\mathcal{E}_{H}\right)=0$. For $j=1 \ldots s$, let

$$
\begin{aligned}
\delta_{j}\left(\mathcal{X}_{j}\right) & :=\frac{\mathrm{d}}{\mathrm{d} t} \mathcal{X}_{j}-\left(A_{j}+B_{j}+C_{j}\right) \mathcal{X}_{j}, \\
\rho_{j}\left(\mathcal{X}_{j}\right) & :=\frac{\mathrm{d}}{\mathrm{d} t} \mathcal{X}_{j}-A_{j} \mathcal{X}_{j} \\
\sigma_{j}\left(\mathcal{X}_{j}\right) & :=\frac{\mathrm{d}}{\mathrm{d} t} \mathcal{X}_{j}-\mathcal{X}_{j} B_{j}-C_{j} \mathcal{X}_{j} .
\end{aligned}
$$

We denote

$$
\begin{aligned}
& H_{0}:=0, \\
& H_{j}:=A_{j}+B_{j}+C_{j}, \quad j \in\{1, \ldots, s\} .
\end{aligned}
$$


Since $A_{j}+B_{j}$ and $A+B$ often occur, we write

$$
D_{j}=A_{j}+B_{j}, \quad D=A+B .
$$

Furthermore, let

$$
\underline{H}_{j}:=H_{1}+\ldots+H_{j-1}, \quad j \in\{1, \ldots, s\} .
$$

We also define a family of iterated commutators by

$$
A_{j}^{[0]}:=A_{j}, \quad B_{j}^{[0]}:=B_{j}, \quad C_{j}^{[0]}:=C_{j},
$$

and recursively for $k \geq 1$,

$$
A_{j}^{[k]}:=\left[A_{j}^{[k-1]}, \underline{H}_{j}\right], \quad B_{j}^{[k]}:=\left[B_{j}^{[k-1]}, \underline{H}_{j}+A_{j}\right], \quad C_{j}^{[k]}:=\left[C_{j}^{[k-1]}, \underline{H}_{j}+H_{j}\right] .
$$

2.2 Order of the local error, order conditions

Via Taylor expansion of the local error operator $\mathcal{L}(t)$, asymptotic order $p$ is characterized by

$$
\frac{\mathrm{d}}{\mathrm{d} t} \mathcal{L}(0)=\frac{\mathrm{d}^{2}}{\mathrm{~d} t^{2}} \mathcal{L}(0)=\ldots=\frac{\mathrm{d}^{p}}{\mathrm{~d} t^{p}} \mathcal{L}(0)=0,
$$

and for a scheme of order $p$ the leading term of the local error is given by

$$
\mathcal{L}_{0}(t):=\frac{t^{p+1}}{(p+1) !} \frac{\mathrm{d}^{p+1}}{\mathrm{~d} t^{p+1}} \mathcal{L}(0)=\mathscr{O}\left(t^{p+1}\right),
$$

such that $\mathcal{L}(t)=\mathcal{L}_{0}(t)+\mathscr{O}\left(t^{p+2}\right)$. According to [9], for a general splitting method of order $p$ the leading term $\mathcal{L}_{0}(t)$ has a special structure, namely

$$
\mathcal{L}_{0}(t)=\text { linear combination of } p \text {-th iterated commutators of } A, B, C,
$$

with coefficients independent of the given problem. As described in [9], this can be used to set up a recursive algorithm for the generation of order conditions. Furthermore, for a given scheme of order $p$, the coefficients in the linear combination (2.10) can then be computed from the conditions for order $p+1$.

In the following, we do not discuss order conditions or the construction of higherorder schemes in detail. In the next section we list some particular schemes as illustrative examples.

2.3 Integral representation of the local error via the defect of the splitting approximation

With the defect ${ }^{1}$

$$
\mathcal{D}=\mathcal{S}^{[1]}=\delta\left(\mathcal{S}^{[0]}\right)=\frac{\mathrm{d}}{\mathrm{d} t} \mathcal{S}^{[0]}-H \mathcal{S}^{[0]}
$$

of the splitting operator $\mathcal{S}^{[0]}$ with respect to the given evolution equation, we obtain a first, basic integral representation of the local error via the variation-of-constant formula,

$$
\mathcal{L}(t)=\int_{0}^{t} \mathcal{E}_{H}(t-\tau) \mathcal{D}(\tau) \mathrm{d} \tau
$$

In Section 5, the integral (2.12) will be expanded into a multiple integral over higherorder defect terms, reflecting the precise order of the scheme.

\footnotetext{
${ }^{1}$ Similarly as $\mathcal{S}=\mathcal{S}^{[0]}, \mathcal{D}=\mathcal{S}^{[1]}$ is a natural and consistent notation for the defect $\mathcal{D}$.
} 
3 Schemes of orders 1, 2, and 4

We list some basic facts about lower-order schemes and their local error structure. These results have been obtained by means of an implementation (in Maple $18^{2}$ ) of the algorithm described in [9].

3.1 First-order Lie-Trotter splitting method

For $s=p=1$ we have

$$
\mathcal{S}=\mathcal{E}_{C} \mathcal{E}_{B} \mathcal{E}_{A}
$$

and the leading term of the local error is

$$
\mathcal{L}_{0}(t)=\frac{t^{2}}{2}([B, A]+[C, A]+[C, B])
$$

\subsection{Second-order Strang splitting method}

Formally, the standard second-order splitting method involves three compositions comprising 5 evaluations of exponentials: For $s=3$, i.e.,

$$
\mathcal{S}=\mathcal{S}_{3} \mathcal{S}_{2} \mathcal{S}_{1}=\mathcal{E}_{C_{3}} \mathcal{E}_{B_{3}} \mathcal{E}_{A_{3}} \cdot \mathcal{E}_{C_{2}} \mathcal{E}_{B_{2}} \mathcal{E}_{A_{2}} \cdot \mathcal{E}_{C_{1}} \mathcal{E}_{B_{1}} \mathcal{E}_{A_{1}},
$$

and $p=2$, the choice of coefficients according to

\begin{tabular}{|rcc|ccc|ccc|}
\hline$a_{1}$ & $b_{1}$ & $c_{1}$ & $a_{2}$ & $b_{2}$ & $c_{2}$ & $a_{3}$ & $b_{3}$ & $c_{3}$ \\
\hline 0 & 0 & $\frac{1}{2}$ & 0 & $\frac{1}{2}$ & 0 & 1 & $\frac{1}{2}$ & $\frac{1}{2}$ \\
\hline
\end{tabular}

yields the symmetric Strang splitting method in the form

$$
\begin{aligned}
\mathcal{S} & =\mathcal{E}_{C / 2} \mathcal{E}_{B / 2} \mathcal{E}_{A} \mathcal{E}_{B / 2} \mathcal{E}_{C / 2} \\
& =\mathcal{E}_{C / 2} \mathcal{E}_{B / 2} \mathcal{E}_{A / 2} \cdot \mathcal{E}_{A / 2} \mathcal{E}_{B / 2} \mathcal{E}_{C / 2}
\end{aligned}
$$

The leading term of the local error reads

$$
\begin{aligned}
\mathcal{L}_{0}(t)=\frac{t^{3}}{6} & \left(\lambda_{1}[A,[A, B]]+\lambda_{2}[A,[A, C]]+\lambda_{3}[B,[A, B]]+\lambda_{4}[C,[A, B]]\right. \\
& \left.+\lambda_{5}[B,[A, C]]+\lambda_{6}[C,[A, C]]+\lambda_{7}[B,[B, C]]+\lambda_{8}[C,[B, C]]\right),
\end{aligned}
$$

with the following coefficients:

\begin{tabular}{|cccccccc|}
\hline$\lambda_{1}$ & $\lambda_{2}$ & $\lambda_{3}$ & $\lambda_{4}$ & $\lambda_{5}$ & $\lambda_{6}$ & $\lambda_{7}$ & $\lambda_{8}$ \\
\hline$\frac{1}{2}$ & $\frac{1}{2}$ & $\frac{1}{4}$ & $-\frac{1}{2}$ & 1 & $\frac{1}{4}$ & $\frac{1}{2}$ & $\frac{1}{4}$ \\
\hline
\end{tabular}

\footnotetext{
2 Maple is a trademark of Maplesoft, Inc.
} 
3.3 Second-order splitting method, complex coefficients with positive real parts

For $s=2$, i.e.,

$$
\mathcal{S}=\mathcal{S}_{2} \cdot \mathcal{S}_{1}=\mathcal{E}_{C_{2}} \mathcal{E}_{B_{2}} \mathcal{E}_{A_{2}} \cdot \mathcal{E}_{C_{1}} \mathcal{E}_{B_{1}} \mathcal{E}_{A_{1}},
$$

and $p=2$ we obtain a pair of complex solutions with positive real parts,

$$
a_{1}=b_{1}=c_{1}=\frac{1}{2}(1-\mathrm{i}), \quad a_{2}=b_{2}=c_{2}=\frac{1}{2}(1+\mathrm{i}),
$$

and

$$
a_{1}=b_{1}=c_{1}=\frac{1}{2}(1+\mathrm{i}), \quad a_{2}=b_{2}=c_{2}=\frac{1}{2}(1-\mathrm{i}) .
$$

The leading term of the local error for $(3.5 \mathrm{a})$ reads

$$
\begin{aligned}
\mathcal{L}_{0}(t)=\frac{t^{3}}{6} & \left(\lambda_{1}[A,[A, B]]+\lambda_{2}[A,[A, C]]+\lambda_{3}[B,[A, B]]+\lambda_{4}[C,[A, B]]\right. \\
& \left.+\lambda_{5}[B,[A, C]]+\lambda_{6}[C,[A, C]]+\lambda_{7}[B,[B, C]]+\lambda_{8}[C,[B, C]]\right),
\end{aligned}
$$

with the following coefficients:

\begin{tabular}{|cccccccc|}
\hline$\lambda_{1}$ & $\lambda_{2}$ & $\lambda_{3}$ & $\lambda_{4}$ & $\lambda_{5}$ & $\lambda_{6}$ & $\lambda_{7}$ & $\lambda_{8}$ \\
\hline$\frac{1}{4}(-1+3 \mathrm{i})$ & $\frac{1}{4}(-1+3 \mathrm{i})$ & $\frac{1}{4}(1+3 \mathrm{i})$ & 1 & $\frac{1}{2}(-1+3 \mathrm{i})$ & $\frac{1}{4}(1+3 \mathrm{i})$ & $\frac{1}{4}(-1+3 \mathrm{i})$ & $\frac{1}{4}(1+3 \mathrm{i})$ \\
\hline
\end{tabular}

3.4 Higher-order splitting methods

Construction by composition A straightforward approach for the construction of higherorder splitting methods relies on composition. For instance, a fourth-order scheme can be constructed by symmetric composition of three steps with $\mathcal{S}=\mathcal{S}(t)$ from (3.3), i.e.,

$$
\mathcal{S}_{\mathrm{comp}}(t)=\mathcal{S}\left(\gamma_{3} t\right) \mathcal{S}\left(\gamma_{2} t\right) \mathcal{S}\left(\gamma_{1} t\right),
$$

and with the 'triple jump' coefficients (see [14])

$$
\begin{aligned}
& \gamma_{1}=\gamma_{3}=\frac{1}{2-2^{1 / 3}} \approx 1.351207191959658, \\
& \gamma_{2}=-\frac{2^{1 / 3}}{2-2^{1 / 3}} \approx-1.702414383919315
\end{aligned}
$$

This results in a symmetric method with 9 compositions and coefficients according to

\begin{tabular}{|rcc|ccc|ccc|}
\hline$a_{1}$ & $b_{1}$ & $c_{1}$ & $a_{2}$ & $b_{2}$ & $c_{2}$ & $a_{3}$ & $b_{3}$ & $c_{3}$ \\
\hline 0 & 0 & $\frac{\gamma_{1}}{2}$ & 0 & $\frac{\gamma_{1}}{2}$ & 0 & $\gamma_{1}$ & $\frac{\gamma_{1}}{2}$ & $\frac{\gamma_{1}}{2}$ \\
\hline \hline$a_{4}$ & $b_{4}$ & $c_{4}$ & $a_{5}$ & $b_{5}$ & $c_{5}$ & $a_{6}$ & $b_{6}$ & $c_{6}$ \\
\hline 0 & 0 & $\frac{\gamma_{2}}{2}$ & 0 & $\frac{\gamma_{2}}{2}$ & 0 & $\gamma_{2}$ & $\frac{\gamma_{2}}{2}$ & $\frac{\gamma_{2}}{2}$ \\
\hline \hline$a_{7}$ & $b_{7}$ & $c_{7}$ & $a_{8}$ & $b_{8}$ & $c_{8}$ & $a_{9}$ & $b_{9}$ & $c_{9}$ \\
\hline 0 & 0 & $\frac{\gamma_{1}}{2}$ & 0 & $\frac{\gamma_{1}}{2}$ & 0 & $\gamma_{1}$ & $\frac{\gamma_{1}}{2}$ & $\frac{\gamma_{1}}{2}$ \\
\hline
\end{tabular}


Since the subflows involving $c_{3}, c_{4}$ and $c_{6}, c_{7}$ can be combined into single subflows involving $c_{3}+c_{4}$ and $c_{6}+c_{7}$, this method requires 13 evaluations of subflows.

For certain applications, in particular parabolic problems, we require (complex) coefficients with positive real parts. This is accomplished by choosing the composition coefficients $\gamma_{j}$ in (3.6a) as complex with real parts, see [15].

For a method of order $p=4$, the leading term of the local error reads

$$
\mathcal{L}_{0}(t)=\frac{t^{5}}{120} \sum_{k=1}^{48} \lambda_{k} K_{k},
$$

with computable coefficients $\lambda_{k}$ and 48 different fourth iterated commutators $K_{k}$ of the given operators $A, B, C$.

Solution of order conditions An alternative strategy to construct higher-order schemes without resorting to composition is based on the solution of the algebraic system of the order conditions, see also [9]. Often the resulting splitting methods turns out to have a smaller error constant than composition schemes. Finding optimized solutions, e.g., by minimizing $\sum_{k} \lambda_{k}^{2}$ in (3.7), is a topic currently under investigation.

\section{Lie-Trotter splitting: Representation of the local error}

In this section we consider the Lie-Trotter scheme and illustrate our strategy for the representation the local error. The (much more intricate) general case is considered in Section 5. We use the notation introduced in Section 2 and make use of some technical results which are collected in $\mathrm{A}$.

Approach We proceed from the local error integral (2.12) involving the defect $\mathcal{D}=\mathcal{S}^{[1]}$, and $\mathcal{S}^{[1]}$ in turn is represented by an integral which is derived from a differential equation of Sylvester type.

For the Lie-Trotter splitting operator (3.1) we write

$$
\mathcal{S}^{[0]}=\mathcal{E}_{C} \mathcal{E}_{B} \cdot \mathcal{E}_{A}=\mathcal{W}^{[0]} \cdot \mathcal{V}^{[0]} .
$$

From

$$
\frac{\mathrm{d}}{\mathrm{d} t} \mathcal{W}^{[0]}=\mathcal{W}^{[0]} B+C \mathcal{W}^{[0]}
$$

we obtain

$$
\frac{\mathrm{d}}{\mathrm{d} t} \mathcal{S}^{[0]}=\frac{\mathrm{d}}{\mathrm{d} t} \mathcal{W}^{[0]} \cdot \mathcal{V}^{[0]}+\mathcal{W}^{[0]} \cdot \frac{\mathrm{d}}{\mathrm{d} t} \mathcal{V}^{[0]}=\left(C \mathcal{W}^{[0]}+\mathcal{W}^{[0]} B\right) \mathcal{V}^{[0]}+\mathcal{W}^{[0]} A \mathcal{V}^{[0]}
$$

and thus,

$$
\frac{\mathrm{d}}{\mathrm{d} t} \mathcal{S}^{[0]}=\left(\mathcal{W}^{[0]} D+C \mathcal{W}^{[0]}\right) \mathcal{V}^{[0]} .
$$

With (4.2), the defect (2.11) can be recast as

$$
\mathcal{S}^{[1]}=\mathcal{W}^{[0]} D \mathcal{V}^{[0]}+C \mathcal{W}^{[0]} \mathcal{V}^{[0]}-(D+C) \mathcal{W}^{[0]} \mathcal{V}^{[0]}=\left[\mathcal{W}^{[0]}, D\right] \mathcal{V}^{[0]}
$$

with $\mathcal{S}^{[1]}(0)=0$. Introducing another shorthand notation,

$$
\mathcal{S}^{[1]}=\mathcal{W}^{[1]} \mathcal{V}^{[0]}, \quad \text { with } \quad \mathcal{W}^{[1]}:=\left[\mathcal{W}^{[0]}, D\right]
$$


where we seek a suitable representation for $\mathcal{W}^{[1]}$. Due to $(4.1)$ and Lemma $5(\mathrm{~A}), \mathcal{W}^{[1]}$ satisfies the inhomogeneous Sylvester equation

$$
\frac{\mathrm{d}}{\mathrm{d} t} \mathcal{W}^{[1]}=\mathcal{W}^{[1]} B+C \mathcal{W}^{[1]}+\sigma\left(\mathcal{W}^{[1]}\right)
$$

with $\mathcal{W}^{[1]}(0)=0$, and

$$
\begin{aligned}
\sigma\left(\mathcal{W}^{[1]}\right) & =\mathcal{W}^{[0]}[B, D]+[C, D] \mathcal{W}^{[0]} \\
& =\mathcal{W}^{[0]}[B, A]+[C, H] \mathcal{W}^{[0]}
\end{aligned}
$$

From (4.5a), by using Lemma A.5(i) we infer the integral representation

$$
\mathcal{W}^{[1]}(t)=\int_{0}^{t} \mathcal{E}_{C}(t-\tau) \sigma\left(\mathcal{W}^{[1]}\right)(\tau) \mathcal{E}_{B}(t-\tau) \mathrm{d} \tau
$$

With $(4.5 \mathrm{~b})$ and $\mathcal{W}^{[0]}=\mathcal{E}_{C} \mathcal{E}_{B}$ this yields

$$
\begin{aligned}
\mathcal{S}^{[1]}(t)=\mathcal{W}^{[1]}(t) \mathcal{V}^{[0]}(t)= & \mathcal{E}_{C}(t) \int_{0}^{t} \mathcal{E}_{B}(t-\tau)[B, A] \mathcal{E}_{B}(\tau) \mathrm{d} \tau \cdot \mathcal{E}_{A}(t) \\
& +\int_{0}^{t} \mathcal{E}_{C}(t-\tau)[C, H] \mathcal{E}_{C}(\tau) \mathrm{d} \tau \cdot \mathcal{E}_{B}(t) \mathcal{E}_{A}(t)
\end{aligned}
$$

Altogether,

$$
\mathcal{L}(t) u=\int_{0}^{t} \mathcal{E}_{H}(t-\tau) \mathcal{S}^{[1]}(\tau) \mathrm{d} \tau \cdot u
$$

Note that $\mathcal{S}^{[1]}(t) u=\mathscr{O}(t)$ and $\mathcal{L}(t) u=\mathscr{O}\left(t^{2}\right)$.

5 General high-order $s$-stage splitting method: Representation of the local error

We assume that the conditions (2.9a) for order $p$ are satisfied. Then,

$$
\mathcal{L}(t) u=\frac{t^{p+1}}{(p+1) !} \frac{\mathrm{d}^{p+1}}{\mathrm{~d} t^{p+1}} \mathcal{L}(0)+\mathscr{O}\left(t^{p+2}\right)
$$

where the leading coefficient $\frac{\mathrm{d}^{p+1}}{\mathrm{~d} t^{p+1}} \mathcal{L}(0)$ is a linear combination of iterated commutators of the data $A, B, C$, see (2.10). Instead of estimating the $\mathscr{O}\left(t^{p+2}\right)$ remainder, we follow a different approach, namely we aim at an exact representation of $\mathcal{L}(t) u$ in the form of a (multiple) integral expansion, generalizing (4.6), (4.7).

In the following we use the notation introduced in Section 2 and make use of some technical results which are collected in A. 
5.1 Representation of of the local error via multiple variation-of-constant

We proceed via generalization of the approach from [13]. Consider the splitting operator

$$
\mathcal{S}^{[0]}=\mathcal{S}_{s}^{[0]} \cdots \mathcal{S}_{1}^{[0]}
$$

with

$$
\mathcal{S}_{j}^{[0]}=\left(\mathcal{E}_{C_{j}} \mathcal{E}_{B_{j}}\right) \mathcal{E}_{A_{j}}=\mathcal{W}_{j}^{[0]} \mathcal{V}_{j}^{[0]}
$$

where $\mathcal{V}_{j}^{[0]}$ and $\mathcal{W}_{j}^{[0]}$ satisfy the initial value problems

$$
\begin{aligned}
\frac{\mathrm{d}}{\mathrm{d} t} \mathcal{V}_{j}^{[0]} & =A_{j} \mathcal{V}_{j}^{[0]}, \quad \mathcal{V}_{j}^{[0]}(0)=\mathcal{I}, \\
\frac{\mathrm{d}}{\mathrm{d} t} \mathcal{W}_{j}^{[0]} & =\mathcal{W}_{j}^{[0]} B_{j}+C_{j} \mathcal{W}_{j}^{[0]}, \quad \mathcal{W}_{j}^{[0]}(0)=\mathcal{I} .
\end{aligned}
$$

With the linear differential operator defined by (2.3), we recursively define the higher-order defect operators $\mathcal{S}^{[q]}$ by

$$
\mathcal{S}^{[q]}:=\delta\left(\mathcal{S}^{[q-1]}\right)=\frac{\mathrm{d}}{\mathrm{d} t} \mathcal{S}^{[q-1]}-H \mathcal{S}^{[q-1]}, \quad q \geq 1
$$

By successive differentiation of (2.12) and evaluation at $t=0$ it can be seen that the order conditions (2.9a) are equivalent to

$$
\mathcal{S}^{[1]}(0)=\frac{\mathrm{d}}{\mathrm{d} t} \mathcal{S}^{[1]}(0)=\ldots=\frac{\mathrm{d}^{p-1}}{\mathrm{~d} t^{p-1}} \mathcal{S}^{[1]}(0)=0,
$$

which in turn is equivalent to

$$
\mathcal{S}^{[1]}(0)=\mathcal{S}^{[2]}(0)=\cdots=\mathcal{S}^{[p]}(0)=0
$$

Hence the local error operator $\mathcal{L}=\mathcal{S}^{[0]}-\mathcal{E}_{H}$ can be expanded via a multiple variationof-constant representation,

$$
\begin{aligned}
\mathcal{L}(t) & =\int_{0}^{t} \mathcal{E}_{H}\left(t-\tau_{1}\right) \mathcal{S}^{[1]}\left(\tau_{1}\right) \mathrm{d} \tau_{1} \\
& =\int_{0}^{t} \int_{0}^{\tau_{1}} \mathcal{E}_{H}\left(t-\tau_{2}\right) \mathcal{S}^{[2]}\left(\tau_{2}\right) \mathrm{d} \tau_{2} \mathrm{~d} \tau_{1} \\
& =\ldots \\
& =\int_{0}^{t} \int_{0}^{\tau_{1}} \cdots \int_{0}^{\tau_{p}} \mathcal{E}_{H}\left(t-\tau_{p+1}\right) \mathcal{S}^{[p+1]}\left(\tau_{p+1}\right) \mathrm{d} \tau_{p+1} \cdots \mathrm{d} \tau_{1} .
\end{aligned}
$$

A slightly different integral representation will be used in Section 5.3 in order to estimate the local error. 
5.2 Recursion for higher-order defect terms

For a rigorous local error analysis, a representation of the higher-order defect operator $\mathcal{S}^{[p+1]}$ appearing in (5.4) is required. This will be obtained by means of a recursion for $\mathcal{S}^{[q]}, q=1,2, \ldots$. Let us illustrate the first step of this procedure for the case $s=3$ :

$$
\begin{aligned}
& \mathcal{S}^{[1]}=\delta\left(\mathcal{S}^{[0]}\right)=\frac{\mathrm{d}}{\mathrm{d} t} \mathcal{S}^{[0]}-H \mathcal{S}^{[0]} \\
& =\frac{\mathrm{d}}{\mathrm{d} t} \mathcal{S}_{3}^{[0]} \cdot \mathcal{S}_{2}^{[0]} \cdot \mathcal{S}_{1}^{[0]}+\mathcal{S}_{3}^{[0]} \cdot \frac{\mathrm{d}}{\mathrm{d} t} \mathcal{S}_{2}^{[0]} \cdot \mathcal{S}_{1}^{[0]}+\mathcal{S}_{3}^{[0]} \cdot \mathcal{S}_{2}^{[0]} \cdot \frac{\mathrm{d}}{\mathrm{d} t} \mathcal{S}_{1}^{[0]} \\
& -\left(H_{3}+H_{2}+H_{1}\right) \mathcal{S}_{3}^{[0]} \mathcal{S}_{2}^{[0]} \mathcal{S}_{1}^{[0]} \\
& =\left(\frac{\mathrm{d}}{\mathrm{d} t} \mathcal{S}_{3}^{[0]}-H_{3} \mathcal{S}_{3}^{[0]}\right) \mathcal{S}_{2}^{[0]} \mathcal{S}_{1}^{[0]} \\
& +\mathcal{S}_{3}^{[0]} \cdot \frac{\mathrm{d}}{\mathrm{d} t} \mathcal{S}_{2}^{[0]} \cdot \mathcal{S}_{1}^{[0]}+\mathcal{S}_{3}^{[0]} \cdot \mathcal{S}_{2}^{[0]} \cdot \frac{\mathrm{d}}{\mathrm{d} t} \mathcal{S}_{1}^{[0]} \\
& -\left(H_{2}+H_{1}\right) \mathcal{S}_{3}^{[0]} \mathcal{S}_{2}^{[0]} \mathcal{S}_{1}^{[0]} \\
& =\left(\frac{\mathrm{d}}{\mathrm{d} t} \mathcal{S}_{3}^{[0]}-H_{3} \mathcal{S}_{3}^{[0]}\right) \mathcal{S}_{2}^{[0]} \mathcal{S}_{1}^{[0]}+\left[\mathcal{S}_{3}^{[0]}, H_{1}+H_{2}\right] \mathcal{S}_{2}^{[0]} \mathcal{S}_{1}^{[0]} \\
& +\mathcal{S}_{3}^{[0]}\left(\frac{\mathrm{d}}{\mathrm{d} t} \mathcal{S}_{2}^{[0]} \cdot \mathcal{S}_{1}^{[0]}+\mathcal{S}_{2}^{[0]} \cdot \frac{\mathrm{d}}{\mathrm{d} t} \mathcal{S}_{1}^{[0]}-\left(H_{2}+H_{1}\right) \mathcal{S}_{2}^{[0]} \mathcal{S}_{1}^{[0]}\right) \\
& =\left(\left(\frac{\mathrm{d}}{\mathrm{d} t} \mathcal{S}_{3}^{[0]}-H_{3} \mathcal{S}_{3}^{[0]}\right)+\left[\mathcal{S}_{3}^{[0]}, H_{1}+H_{2}\right]\right) \mathcal{S}_{2}^{[0]} \mathcal{S}_{1}^{[0]} \\
& +\mathcal{S}_{3}^{[0]}\left(\left(\frac{\mathrm{d}}{\mathrm{d} t} \mathcal{S}_{2}^{[0]}-H_{2} \mathcal{S}_{2}^{[0]}\right)+\left[\mathcal{S}_{2}^{[0]}, H_{1}\right]\right) \mathcal{S}_{1}^{[0]} \\
& +\mathcal{S}_{3}^{[0]} \mathcal{S}_{2}^{[0]}\left(\frac{\mathrm{d}}{\mathrm{d} t} \mathcal{S}_{1}^{[0]}-H_{1} \mathcal{S}_{1}^{[0]}\right) \\
& =\left(\left[\mathcal{S}_{3}^{[0]}, \underline{H}_{3}\right]+\delta_{3}\left(\mathcal{S}_{3}^{[0]}\right)\right) \mathcal{S}_{2}^{[0]} \mathcal{S}_{1}^{[0]} \\
& +\mathcal{S}_{3}^{[0]}\left(\left[\mathcal{S}_{2}^{[0]}, \underline{H}_{2}\right]+\delta_{2}\left(\mathcal{S}_{2}^{[0]}\right)\right) \mathcal{S}_{1}^{[0]} \\
& +\mathcal{S}_{3}^{[0]} \mathcal{S}_{2}^{[0]}\left(\left[\mathcal{S}_{1}^{[0]}, \underline{H}_{1}\right]+\delta_{1}\left(\mathcal{S}_{1}^{[0]}\right)\right) \\
& =: \mathcal{S}_{3}^{[1]} \mathcal{S}_{2}^{[0]} \mathcal{S}_{1}^{[0]}+\mathcal{S}_{3}^{[0]} \mathcal{S}_{2}^{[1]} \mathcal{S}_{1}^{[0]}+\mathcal{S}_{3}^{[0]} \mathcal{S}_{2}^{[0]} \mathcal{S}_{1}^{[1]} \text {, }
\end{aligned}
$$

with $\delta_{j}(\cdot)$ from $(2.4 \mathrm{a})$ and $\underline{H}_{j}$ from (2.7). This representation is independent of the particular structure of $\mathcal{S}_{j}^{[0]}$, and the general pattern is clearly visible. Moreover, with the recursive definition ${ }^{3}$

$$
\mathcal{S}_{j}^{[k+1]}:=\left[\mathcal{S}_{j}^{[k]}, \underline{H}_{j}\right]+\delta_{j}\left(\mathcal{S}_{j}^{[k]}\right), \quad k \geq 0,
$$

this generalizes in the same way as in [13] in form of the following Leibniz-type formula.

Lemma 1 The higher-order defect operators $\mathcal{S}^{[q]}$ defined by (5.2) admit a representation in the form of a multinomial expansion,

$$
\mathcal{S}^{[q]}=\sum_{k \in \mathbb{N}_{0}^{s},|k|=q}\left(\begin{array}{l}
q \\
k
\end{array}\right) \mathcal{S}_{s}^{\left[k_{s}\right]} \ldots \mathcal{S}_{1}^{\left[k_{1}\right]}, \quad q \geq 0,
$$

where the $\mathcal{S}_{j}^{[k]}$ are recursively defined by (5.5).

Proof Analogously to [13, Lemma 5].

\footnotetext{
${ }^{3}$ Note that the mapping $\mathcal{S}_{j}^{[q]} \mapsto \mathcal{S}_{j}^{[q+1]}$ is linear. For $A=0$, the definition of $\mathcal{S}_{j}^{[q+1]}$ in (5.5) is equivalent to that from [13].
} 
With $\rho_{j}(\cdot), \sigma_{j}(\cdot)$ from $(2.4)$ and from $(5.1)$ we have $\rho_{j}\left(\mathcal{V}^{[j]}\right)=0$ and $\sigma_{j}\left(\mathcal{W}^{[j]}\right)=0$. Furthermore, we recursively define

$$
\begin{aligned}
\mathcal{V}_{j}^{[k+1]} & :=\left[\mathcal{V}_{j}^{[k]}, \underline{H}_{j}\right]+\rho_{j}\left(\mathcal{V}_{j}^{[k]}\right), \quad k \geq 0, \\
\mathcal{W}_{j}^{[k+1]} & :=\left[\mathcal{W}_{j}^{[k]}, \underline{H}_{j}+D_{j}\right]+\sigma_{j}\left(\mathcal{W}_{j}^{[k]}\right), \quad k \geq 0,
\end{aligned}
$$

with $D_{j}$ from (2.6) and $\underline{H}_{j}$ from (2.7). $\mathcal{V}_{j}^{[k]}$ and $\mathcal{W}_{j}^{[k]}$ are building blocks for $\mathcal{S}_{j}^{[k]}$ :

Lemma 2 The higher-order defect terms $\mathcal{S}_{j}^{[k]}$ defined by (5.5) admit a representation in form of a binomial expansion,

$$
\mathcal{S}_{j}^{[k]}=\sum_{\ell=0}^{k}\left(\begin{array}{l}
k \\
\ell
\end{array}\right) \mathcal{W}_{j}^{[k-\ell]} \mathcal{V}_{j}^{[\ell]}, \quad k \geq 0, \quad j \in\{1, \ldots, s\}
$$

with $\mathcal{V}_{j}^{[\ell]}$ and $\mathcal{W}_{j}^{[\ell]}$ defined by $(5.7)$

Proof See Appendix B.

Finally, we provide recursive representations for the $\mathcal{V}_{j}^{[k]}$ and $\mathcal{W}_{j}^{[k]}$.

\section{Lemma 3}

(i) The operators $\mathcal{V}_{j}^{[k]}$ defined by (5.7a) satisfy

$$
\rho_{j}\left(\mathcal{V}_{j}^{[k]}\right)=\sum_{\ell=1}^{k}\left(\begin{array}{l}
k \\
\ell
\end{array}\right) A_{j}^{[\ell]} \mathcal{V}_{j}^{[k-\ell]}, \quad k \geq 0, \quad j \in\{1, \ldots, s\},
$$

with $A_{j}^{[\ell]}$ defined in (2.8) and $\rho_{j}(\cdot)$ from $(2.4 \mathrm{~b})$. That is, $\mathcal{V}_{j}^{[k]}$ satisfy the (recursively defined) evolution equations

$$
\frac{\mathrm{d}}{\mathrm{d} t} \mathcal{V}_{j}^{[k]}=A_{j} \mathcal{V}_{j}^{[k]}+\sum_{\ell=1}^{k}\left(\begin{array}{l}
k \\
\ell
\end{array}\right) A_{j}^{[\ell]} \mathcal{V}_{j}^{[k-\ell]}
$$

Thus,

$$
\begin{aligned}
\mathcal{V}_{j}^{[k]}(t) & =\mathcal{E}_{A_{j}}(t) \mathcal{V}_{j}^{[k]}(0)+\int_{0}^{t} \mathcal{E}_{A_{j}}(t-\tau) \rho_{j}\left(\mathcal{V}_{j}^{[k]}\right)(\tau) \mathrm{d} \tau \\
& =: \mathcal{V}_{j, 0}^{[k]}(t)+\mathcal{V}_{j, 1}^{[k]}(t)
\end{aligned}
$$

with initial value $\mathcal{V}_{j}^{[k]}(0)=\left[\mathcal{V}_{j}^{[k-1]}(0), \underline{H}_{j}\right]+\rho_{j}\left(\mathcal{V}_{j}^{[k-1]}\right)(0)$, and $\rho_{j}\left(\mathcal{V}_{j}^{[k]}\right)$ from (5.9a).

(ii) The $\mathcal{W}_{j}^{[k]}$ defined by (5.7b) satisfy

$$
\sigma_{j}\left(\mathcal{W}_{j}^{[k]}\right)=\sum_{\ell=1}^{k}\left(\begin{array}{l}
k \\
\ell
\end{array}\right)\left(\mathcal{W}_{j}^{[k-\ell]} B_{j}^{[\ell]}+C_{j}^{[\ell]} \mathcal{W}_{j}^{[k-\ell]}\right), \quad k \geq 0, \quad j \in\{1, \ldots, s\},
$$

\footnotetext{
${ }^{4}$ Special case $k=0: \mathcal{V}_{j}^{[0]}(0)=\mathcal{I}$.
} 
with $B_{j}^{[\ell]}$ and $C_{j}^{[\ell]}$ defined in (2.8) and with $\sigma_{j}(\cdot)$ from $(2.4 \mathrm{c})$. That is, $\mathcal{W}_{j}^{[k]}$ satisfy the (recursively defined) evolution equations of Sylvester type,

$$
\frac{\mathrm{d}}{\mathrm{d} t} \mathcal{W}_{j}^{[k]}=\mathcal{W}_{j}^{[k]} B_{j}+C_{j} \mathcal{W}_{j}^{[k]}+\sum_{\ell=1}^{k}\left(\begin{array}{l}
k \\
\ell
\end{array}\right)\left(\mathcal{W}_{j}^{[k-\ell]} B_{j}^{[\ell]}+C_{j}^{[\ell]} \mathcal{W}_{j}^{[k-\ell]}\right)
$$

Thus, due to (A.6),

$$
\begin{aligned}
\mathcal{W}_{j}^{[k]}(t) & =\mathcal{E}_{C_{j}}(t) \mathcal{W}_{j}^{[k]}(0) \mathcal{E}_{B_{j}}(t)+\int_{0}^{t} \mathcal{E}_{C_{j}}(t-\tau) \sigma_{j}\left(\mathcal{W}_{j}^{[k]}\right)(\tau) \mathcal{E}_{B_{j}}(t-\tau) \mathrm{d} \tau \\
& =: \mathcal{W}_{j, 0}^{[k]}(t)+\mathcal{W}_{j, 1}^{[k]}(t),
\end{aligned}
$$

with initial value $\mathcal{W}_{j}^{[k]}(0)=\left[\mathcal{W}_{j}^{[k-1]}(0), \underline{H}_{j}+D_{j}\right]+\sigma_{j}\left(\mathcal{W}_{j}^{[k-1]}\right)(0)$, and $\sigma_{j}\left(\mathcal{W}_{j}^{[k]}\right)$ from (5.10a).

Proof See Appendix B.

\subsection{Expansion and estimation of the local error}

Assuming the conditions (2.9a) for order $p$ to be satisfied, which are equivalent to $(5.3 \mathrm{~b})$, the local error can now be expanded, in a way analogous to [13]. Assume first that the conditions $\mathcal{S}^{[1]}(0)=\ldots=\mathcal{S}^{[p-1]}(0)=0$ for order $p-1$ are satisfied. Then the local error can be represented in the form

$$
\mathcal{L}(t)=\int_{0}^{t} \cdots \int_{0}^{\tau_{p-1}} \mathcal{E}_{H}\left(t-\tau_{p}\right) \mathcal{S}^{[p]}\left(\tau_{p}\right) \mathrm{d} \tau_{p} \cdots \mathrm{d} \tau_{1}
$$

From Lemma 1 and Lemma 2 we have

$$
\mathcal{S}^{[p]}=\sum_{k \in \mathbb{N}_{0}^{s},|k|=p}\left(\begin{array}{l}
p \\
k
\end{array}\right) \mathcal{S}_{s}^{\left[k_{s}\right]} \cdots \mathcal{S}_{1}^{\left[k_{1}\right]}
$$

with

$$
\mathcal{S}_{j}^{[k]}=\sum_{\ell=0}^{k}\left(\begin{array}{l}
k \\
\ell
\end{array}\right) \mathcal{W}_{j}^{[k-\ell]} \mathcal{V}_{j}^{[\ell]}, \quad j \in\{1, \ldots, s\} .
$$

$\mathcal{V}_{j}^{[k]}=\mathcal{V}_{j, 0}^{[k]}+\mathcal{V}_{j, 1}^{[k]}$ and $\mathcal{W}_{j}^{[k]}=\mathcal{W}_{j, 0}^{[k]}+\mathcal{W}_{j, 1}^{[k]}$ satisfy recursively defined integral representations as specified in Lemma $3,(5.9 \mathrm{c})$ and $(5.10 \mathrm{c})$. Note that $\mathcal{V}_{j, 0}^{[k]}(t)=\mathscr{O}(1)$,

${ }^{5}$ Special case $k=0: \mathcal{W}_{j}^{[0]}(0)=\mathcal{I}$. 
$\mathcal{W}_{j, 0}^{[k]}(t)=\mathscr{O}(1)$, and $\mathcal{V}_{j, 1}^{[k]}(t)=\mathscr{O}(t), \mathcal{V}_{j, 1}^{[k]}(t)=\mathscr{O}(t)$. We now write $\mathcal{S}^{[p]}$ in the form

$$
\begin{aligned}
\mathcal{S}^{[p]}= & \sum_{k \in \mathbb{N}_{0}^{s},|k|=p}\left(\begin{array}{l}
p \\
k
\end{array}\right) \prod_{j=1}^{s} \sum_{\ell_{j}=0}^{k_{j}}\left(\begin{array}{c}
k_{j} \\
\ell_{j}
\end{array}\right) \mathcal{W}_{j}^{\left[k_{j}-\ell_{j}\right]} \mathcal{V}_{j}^{\left[\ell_{j}\right]} \\
= & \sum_{k \in \mathbb{N}_{0}^{s},|k|=p}\left(\begin{array}{l}
p \\
k
\end{array}\right) \prod_{j=1}^{s} \sum_{\ell_{j}=0}^{k_{j}}\left(\begin{array}{l}
k_{j} \\
\ell_{j}
\end{array}\right) \mathcal{W}_{j, 0}^{\left[k_{j}-\ell_{j}\right]} \mathcal{V}_{j, 0}^{\left[\ell_{j}\right]} \\
& +\sum_{k \in \mathbb{N}_{0}^{s},|k|=p}\left(\begin{array}{l}
p \\
k
\end{array}\right) \prod_{j=1}^{s} \sum_{\ell_{j}=0}^{k_{j}}\left(\begin{array}{l}
k_{j} \\
\ell_{j}
\end{array}\right) \\
& \cdot\left(\mathcal{W}_{j, 0}^{\left[k_{j}-\ell_{j}\right]} \mathcal{V}_{j, 1}^{\left[\ell_{j}\right]}+\mathcal{W}_{j, 1}^{\left[k_{j}-\ell_{j}\right]} \mathcal{V}_{j, 0}^{\left[\ell_{j}\right]}+\mathcal{W}_{j, 1}^{\left[k_{j}-\ell_{j}\right]} \mathcal{V}_{j, 1}^{\left[\ell_{j}\right]}\right)
\end{aligned}
$$

Here, the first sum (5.11a) equals $\mathcal{Q}=\mathcal{Q}(t)$, where

$$
\mathcal{Q}(t)=\sum_{k \in \mathbb{N}_{0}^{s},|k|=p}\left(\begin{array}{l}
p \\
k
\end{array}\right) \prod_{j=1}^{s} \sum_{\ell_{j}=0}^{k_{j}}\left(\begin{array}{l}
k_{j} \\
\ell_{j}
\end{array}\right) \mathcal{E}_{C_{j}}(t) \mathcal{W}_{j}^{\left[k_{j}-\ell_{j}\right]}(0) \mathcal{E}_{B_{j}}(t) \mathcal{E}_{A_{j}}(t) \mathcal{V}_{j}^{\left[\ell_{j}\right]}(0)
$$

and the second sum (5.11b) is $\mathscr{O}(t)$. Now assuming that the $p$-th order condition $\mathcal{S}^{[p]}(0)=0$ is also satisfied, we obtain

$$
\mathcal{S}^{[p]}(0)=\mathcal{Q}(0)=\sum_{k \in \mathbb{N}_{0}^{s},|k|=p}\left(\begin{array}{l}
p \\
k
\end{array}\right) \prod_{j=1}^{s} \sum_{\ell_{j}=0}^{k_{j}}\left(\begin{array}{l}
k_{j} \\
\ell_{j}
\end{array}\right) \mathcal{W}_{j}^{\left[k_{j}-\ell_{j}\right]}(0) \mathcal{V}_{j}^{\left[\ell_{j}\right]}(0)=0
$$

Thus,

$$
\mathcal{Q}(t)=\mathcal{Q}(t)-\mathcal{Q}(0)=\sum_{k \in \mathbb{N}_{0}^{s},|k|=p}\left(\begin{array}{l}
p \\
k
\end{array}\right) \prod_{j=1}^{s} \sum_{\ell_{j}=0}^{k_{j}}\left(\begin{array}{l}
k_{j} \\
\ell_{j}
\end{array}\right) \mathcal{R}_{k_{j}, \ell_{j}}(t)
$$

with

$$
\begin{aligned}
\mathcal{R}_{k_{j}, \ell_{j}}(t)= & \mathcal{E}_{C_{j}}(t) \mathcal{W}_{j}^{\left[k_{j}-\ell_{j}\right]}(0) \mathcal{E}_{B_{j}}(t) \mathcal{E}_{A_{j}}(t) \mathcal{V}_{j}^{\left[\ell_{j}\right]}(0)-\mathcal{W}_{j}^{\left[k_{j}-\ell_{j}\right]}(0) \mathcal{V}_{j}^{\left[\ell_{j}\right]}(0) \\
= & \left(\mathcal{E}_{C_{j}}(t)-\mathcal{I}\right) \mathcal{W}_{j}^{\left[k_{j}-\ell_{j}\right]}(0) \mathcal{E}_{B_{j}}(t) \mathcal{E}_{A_{j}}(t) \mathcal{V}_{j}^{\left[\ell_{j}\right]}(0) \\
& +\mathcal{W}_{j}^{\left[k_{j}-\ell_{j}\right]}(0)\left(\mathcal{E}_{B_{j}}(t) \mathcal{E}_{A_{j}}(t)-\mathcal{I}\right) \mathcal{V}_{j}^{\left[\ell_{j}\right]}(0)
\end{aligned}
$$

Here,

$$
\begin{aligned}
\mathcal{E}_{C_{j}}(t)-\mathcal{I} & =\mathscr{O}(t), \\
\mathcal{E}_{B_{j}}(t) \mathcal{E}_{A_{j}}(t)-\mathcal{I} & =\left(\mathcal{E}_{B_{j}}(t) \mathcal{E}_{A_{j}}(t)-\mathcal{E}_{A_{j}+B_{j}}(t)\right)+\left(\mathcal{E}_{A_{j}+B_{j}}(t)-\mathcal{I}\right)=\mathscr{O}(t) .
\end{aligned}
$$

Altogether, this shows $\mathcal{S}^{[p]}(t)=\mathscr{O}(t)$. The resulting representation for the local error cannot be expressed in an explicit way, but rather it is defined algorithmically by the process described above. We conclude: 
Proposition 1 For a scheme of asymptotic order p, i.e., under assumption (2.9a), the local error $\mathcal{L}(t) u=\mathcal{S}(t) u-\mathcal{E}_{H}(t) u$ satisfies

$$
\mathcal{L}(t) u=\mathscr{O}\left(t^{p+1}\right)
$$

Here $\mathscr{O}\left(t^{p+1}\right)$ means $\mathcal{C}(t) \cdot t^{p+1}$, where $\mathcal{C}(t)$ is well-defined and bounded provided that all expressions occurring in the recursive representation of $\mathcal{L}(t)$ are well-defined and bounded when $\mathcal{L}(t)$ is applied to $u$.

In practical applications this implies certain regularity requirements on $u$, in particular due to the presence of the iterated commutators $(2.8 \mathrm{~b})$ in the representation for $\mathcal{L}(t)$; see in particular Lemma 3 .

Remark 1 For the case of splitting into two operators (with $A=0$ ), we have $\mathcal{S}_{j}^{[k]} \equiv$ $\mathcal{W}_{j}^{[k]}$, and the results from [13] are obtained as a special case. For $B=0$ or $C=0$, an alternative local error representation, different from [13], is obtained.

The considered above suggest itself that the case of splitting into more that three operators can be treated in s similar way, e.g., for $H=H_{I}+H_{I I}+H_{I I I}+H_{I V}$, with $\mathcal{V}_{j}=\mathcal{E}_{H_{I I, j}} \mathcal{E}_{H_{I, j}}$ and $\mathcal{W}_{j}=\mathcal{E}_{H_{I V, j}} \mathcal{E}_{H_{I I I, j}}$, and an appropriately richer combinatorial structure. However, we refrain from going into more details to avoid abounding technicalities in this paper.

\section{Defect-based a posteriori error estimators}

For practical purposes, according to [13], we aim for an estimate of the local error $\mathcal{L}(t) u$ by a practically computable local error estimator $\mathcal{P}(t) u$. In principle, one may use the computable leading term in the Taylor expansion of $\mathcal{L}(t) u$,

$$
\mathcal{P}(t) u=\mathcal{L}_{0}(t) u=\frac{t^{p+1}}{(p+1) !} \frac{\mathrm{d}^{p+1}}{\mathrm{~d} t^{p+1}} \mathcal{L}(0) u,
$$

see $(2.9 \mathrm{~b})$. However, this complex object (see for instance (3.7)) and can only be evaluated at a high effort. Therefore we follow the alternative approach which has been promoted in [13]. Consider the local error integral (2.12) and denote the integrand by $f(\tau)$ (for fixed $t$ ). For a scheme of order $p$ we have $f^{(0)}(0)=f^{(1)}(0)=\ldots=f^{(p-1)}(0)=0$. Via Taylor expansion, this suggests the approximation

$$
\int_{0}^{t} f(\tau) \mathrm{d} \tau \approx \int_{0}^{t} \frac{\tau^{p}}{p !} f^{(p)}(0) \mathrm{d} \tau=\frac{t^{p+1}}{(p+1) !} f^{(p)}(0) \approx \frac{t}{p+1} f(t),
$$

with an expected approximation error of order $\mathscr{O}\left(t^{p+2}\right)$. This can also be interpreted as an Hermite quadrature approximation to the integral over $f$, see [13].

Thus we consider the local error estimator

$$
\mathcal{P}(t) u=\frac{t}{p+1} \mathcal{D}(t) u=\frac{t}{p+1}\left(\frac{\mathrm{d}}{\mathrm{d} t} \mathcal{S}(t)-H \mathcal{S}(t)\right) u .
$$

We refer to Section 7 for the practical, $t$-derivative-free evaluation of $\mathcal{D}(t) u$.

In the following we show that this estimator is indeed asymptotically correct, i.e., the deviation $\mathcal{P}(t) u-\mathcal{L}(t) u$ is one order higher than $\mathcal{L}(t) u$, see Proposition 2. To this end, a detailed representation of the deviation is deduced in several steps, where we suppress the argument $u$ for brevity. 
Peano representation of the quadrature error We consider the local error integral (2.12), employing the notation from (4.7),

$$
\mathcal{L}(t)=\int_{0}^{t} \mathcal{F}(\tau ; t) \mathrm{d} \tau, \quad \text { with integrand } \quad \mathcal{F}(\tau ; t)=\mathcal{E}_{H}(t-\tau) \mathcal{S}^{[1]}(\tau) .
$$

The $(p+1)$-st order Peano representation for the quadrature error reads

$$
\mathcal{P}(t)-\mathcal{L}(t)=\int_{0}^{t} K_{p+1}(\tau ; t) \frac{\partial^{p+1}}{\partial \tau^{p+1}} \mathcal{F}(\tau ; t) \mathrm{d} \tau,
$$

with kernel

$$
K_{p+1}(\tau ; t)=\frac{\tau(t-\tau)^{p}}{(p+1) !} .
$$

Integrating by parts we obtain the $p$-th order Peano representation

$$
\mathcal{P}(t)-\mathcal{L}(t)=\int_{0}^{t} K_{p}(\tau ; t) \frac{\partial^{p}}{\partial \tau^{p}} \mathcal{F}(\tau ; t) \mathrm{d} \tau
$$

with kernel

$$
K_{p}(\tau ; t)=\frac{(t-(p+1) \tau)(t-\tau)^{p-1}}{(p+1) !}, \quad \int_{0}^{t} K_{p}(\tau ; t) \mathrm{d} \tau=0 .
$$

Estimation of the quadrature error The analysis comprises three steps.

(i) Making use of $(5.3 \mathrm{~b})$ yields

$$
\mathcal{P}(t)-\mathcal{L}(t)=\int_{0}^{t} K_{p}(\tau ; t) \mathcal{E}_{H}(t-\tau) \mathcal{S}^{[p+1]}(\tau) \mathrm{d} \tau,
$$

which can be rewritten as (see $(6.3 \mathrm{~b}))$

$$
\begin{aligned}
\mathcal{P}(t)-\mathcal{L}(t)= & \underbrace{\int_{0}^{t} K_{p}(\tau ; t) \mathrm{d} \tau}_{=0} \cdot \mathcal{E}_{H}(t) \mathcal{S}^{[p+1]}(0) \\
& +\int_{0}^{t} K_{p}(\tau ; t) \mathcal{E}_{H}(t-\tau)\left(\mathcal{S}^{[p+1]}(\tau)-\mathcal{E}_{H}(\tau) \mathcal{S}^{[p+1]}(0)\right) \mathrm{d} \tau
\end{aligned}
$$

Since $K_{p}(\tau ; t)=\mathscr{O}\left(\tau^{p}\right)$ for $0 \leq \tau \leq t$, it remains to be shown that

$$
\mathcal{S}^{[p+1]}(t)-\mathcal{E}_{H}(t) \mathcal{S}^{[p+1]}(0)=\mathscr{O}(t) .
$$

(ii) For a method of order $p, \mathcal{S}^{[p+1]}(0)$ is a linear combination of iterated commutators involving the operators $A, B, C$ (see [9]). It also satisfies

$$
\mathcal{S}^{[p+1]}(0)=\sum_{k \in \mathbb{N}_{0}^{s},|k|=p+1}\left(\begin{array}{c}
p+1 \\
k
\end{array}\right) \mathcal{S}_{s}^{\left[k_{s}\right]}(0) \cdots \mathcal{S}_{1}^{\left[k_{1}\right]}(0),
$$

where

$$
\mathcal{S}_{j}^{[k]}(0)=\sum_{\ell=0}^{k}\left(\begin{array}{l}
k \\
\ell
\end{array}\right) \mathcal{W}_{j}^{[k-\ell]}(0) \mathcal{V}_{j}^{[\ell]}(0), \quad k \geq 0, \quad j \in\{1, \ldots, s\},
$$


with $\mathcal{V}_{j}^{[\ell]}$ and $\mathcal{W}_{j}^{[\ell]}$ defined by (5.7); see Lemma 1 and Lemma 2.

Consider the auxiliary quantity

$$
\widetilde{\mathcal{S}}^{[p+1]}(t)=\sum_{k \in \mathbb{N}_{0}^{s},|k|=p+1}\left(\begin{array}{c}
p+1 \\
k
\end{array}\right) \widetilde{\mathcal{S}}_{s}^{\left[k_{s}\right]}(t) \cdots \widetilde{\mathcal{S}}_{1}^{\left[k_{1}\right]}(t),
$$

with

$$
\widetilde{\mathcal{S}}_{j}^{[k]}=\sum_{\ell=0}^{k}\left(\begin{array}{l}
k \\
\ell
\end{array}\right) \widetilde{\mathcal{W}}_{j}^{[k-\ell]} \widetilde{\mathcal{V}}_{j}^{[\ell]}, \quad k \geq 0, \quad j \in\{1, \ldots, s\},
$$

where $\widetilde{\mathcal{V}}_{j}^{[k]}$ and $\widetilde{\mathcal{W}}_{j}^{[k-\ell]}$ are the $\mathscr{O}(1)$ contributions to $\mathcal{V}_{j}^{[k]}$ and $\mathcal{W}_{j}^{[k-\ell]}$, respectively $($ see $(5.9 \mathrm{c})(5.10 \mathrm{c}))$, i.e.,

$$
\begin{aligned}
\widetilde{\mathcal{V}}_{j}^{[k]}(t) & =\mathcal{V}_{j, 0}^{[k]}(t)=\mathcal{E}_{A_{j}}(t) \mathcal{V}_{j}^{[k]}(0), \\
\widetilde{\mathcal{W}}_{j}^{[k]}(t) & =\mathcal{W}_{j, 0}^{[k]}(t)=\mathcal{E}_{C_{j}}(t) \mathcal{W}_{j}^{[k]}(0) \mathcal{E}_{B_{j}}(t),
\end{aligned}
$$

satisfying

$$
\begin{aligned}
\mathrm{d} d & \widetilde{\mathcal{V}}_{j}^{[k]}=A_{j} \widetilde{\mathcal{V}}_{j}^{[k]}, \quad \text { i.e., } \quad \rho_{j}\left(\widetilde{\mathcal{V}}_{j}^{[k]}\right)=0, \\
\mathrm{~d} t \widetilde{\mathcal{W}}_{j}^{[k]} & =\widetilde{\mathcal{W}}_{j}^{[k]} B_{j}+C_{j} \widetilde{\mathcal{W}}_{j}^{[k]}, \quad \text { i.e., } \quad \sigma_{j}\left(\widetilde{\mathcal{W}}_{j}^{[k]}\right)=0 .
\end{aligned}
$$

In the current step (ii) the aim is to estimate the difference $\widetilde{\mathcal{S}}^{[p+1]}(t)-\mathcal{E}_{H}(t) \mathcal{S}^{[p+1]}(0)$. To this end we note that the $\widetilde{\mathcal{S}}_{j}^{[k]}$ from $(6.7 \mathrm{~b})$ have a structure identical to the $\mathcal{S}_{j}^{[k]}$ (see Lemma 2), with $\mathcal{V}_{j}^{[k]} \mathcal{W}_{j}^{[k-\ell]}$ replaced by $\widetilde{\mathcal{V}}_{j}^{[k]} \widetilde{\mathcal{W}}_{j}^{[k-\ell]}$. The terms in the $\operatorname{sum}(6.7 a)$,

$$
\widetilde{\mathcal{S}}_{s}^{\left[k_{s}\right]}(t) \cdots \widetilde{\mathcal{S}}_{1}^{\left[k_{1}\right]}(t),
$$

are splitting analogues of

$$
\mathcal{E}_{H}(t) \mathcal{S}_{s}^{\left[k_{s}\right]}(0) \cdots \mathcal{S}_{1}^{\left[k_{1}\right]}(0),
$$

and $(6.8 \mathrm{a}),(6.8 \mathrm{~b})$ have identical initial values $\mathcal{S}_{s}^{\left[k_{s}\right]}(0) \cdots \mathcal{S}_{1}^{\left[k_{1}\right]}(0)$. In order to estimate the difference between $(6.8 \mathrm{a})$ and $(6.8 \mathrm{~b})$ for $t>0$ we compute, analogously as in Section 5.2,

$$
\begin{aligned}
\delta\left(\widetilde{\mathcal{S}}_{s}^{\left[k_{s}\right]} \ldots \widetilde{\mathcal{S}}_{1}^{\left[k_{1}\right]}\right) & =\frac{\mathrm{d}}{\mathrm{d} t}\left(\widetilde{\mathcal{S}}_{s}^{\left[k_{s}\right]} \ldots \widetilde{\mathcal{S}}_{1}^{\left[k_{1}\right]}\right)-H \cdot \widetilde{\mathcal{S}}_{s}^{\left[k_{s}\right]} \ldots \widetilde{\mathcal{S}}_{1}^{\left[k_{1}\right]} \\
& =\sum_{\ell \in \mathbb{N}_{0}^{s},|\ell|=1} \widetilde{\mathcal{S}}_{s}^{\left[k_{s} ; \ell_{s}\right]} \ldots \widetilde{\mathcal{S}}_{1}^{\left[k_{1} ; \ell_{1}\right]}
\end{aligned}
$$

with $\widetilde{\mathcal{S}}_{j}^{[k ; 0]}=\widetilde{\mathcal{S}}_{j}^{[k]}$ from $(6.7 \mathrm{~b})$ and where, by a routine calculation as in the proof of Lemma 2,

$$
\begin{aligned}
\widetilde{\mathcal{S}}_{j}^{[k ; 1]} & =\left[\widetilde{\mathcal{S}}_{j}^{[k]}, \underline{H}_{j}\right]+\delta_{j}\left(\widetilde{\mathcal{S}}_{j}^{[k]}\right) \\
& =\sum_{\ell=0}^{k}\left(\begin{array}{l}
k \\
\ell
\end{array}\right)\left(\widetilde{\mathcal{W}}_{j}^{[k-\ell]}\left[\widetilde{\mathcal{V}}_{j}^{[\ell]}, \underline{H}_{j}\right]+\left[\widetilde{\mathcal{W}}_{j}^{[k-\ell]}, \underline{H}_{j}+D_{j}\right] \widetilde{\mathcal{V}}_{j}^{[\ell]}\right) .
\end{aligned}
$$


Due to Lemma 4 and Lemma 5 (A), the terms $\left[\widetilde{\mathcal{V}}_{j}^{[\ell]}, \underline{H}_{j}\right]$ and $\left[\widetilde{\mathcal{W}}_{j}^{[k-\ell]}, \underline{H}_{j}+D_{j}\right]$ are solutions to the evolution equations

$$
\left\{\begin{array}{l}
\frac{\mathrm{d}}{\mathrm{d} t}\left[\widetilde{\mathcal{V}}_{j}^{[\ell]}, \underline{H}_{j}\right]=A_{j}\left[\widetilde{\mathcal{V}}_{j}^{[\ell]}, \underline{H}_{j}\right]+A_{j}^{[1]} \widetilde{\mathcal{V}}_{j}^{[\ell]} \\
\widetilde{\mathcal{V}}_{j}^{[\ell]}(0)=\mathcal{V}_{j}^{[\ell]}(0)
\end{array}\right.
$$

and

$$
\left\{\begin{aligned}
\left.\frac{\mathrm{d}}{\mathrm{d} t} \widetilde{\mathcal{W}}_{j}^{[k-\ell]}, \underline{H}_{j}+D_{j}\right]= & {\left[\widetilde{\mathcal{W}}_{j}^{[k-\ell]}, \underline{H}_{j}+D_{j}\right] B_{j}+C_{j}\left[\widetilde{\mathcal{W}}_{j}^{[k-\ell]}, \underline{H}_{j}+D_{j}\right] } \\
& +\widetilde{\mathcal{W}}_{j}^{[k-\ell]} B_{j}^{[1]}+C_{j}^{[1]} \widetilde{\mathcal{W}}_{j}^{[k-\ell]}, \\
\widetilde{\mathcal{W}}_{j}^{[\ell]}(0)=\mathcal{W}_{j}^{[\ell]}(0), &
\end{aligned}\right.
$$

with corresponding integral representations. Collecting all these preliminary considerations we obtain

$$
\left.\delta\left(\widetilde{\mathcal{S}}_{s}^{\left[k_{s}\right]} \ldots \widetilde{\mathcal{S}}_{1}^{\left[k_{1}\right]}\right)-\mathcal{E}_{H}(t) \mathcal{S}_{s}^{\left[k_{s}\right]}(0) \cdots \mathcal{S}_{1}^{\left[k_{1}\right]}(0)\right)=\mathscr{O}(1),
$$

with homogeneous initial condition. Consequently, the difference between (6.8a) and $(6.8 \mathrm{~b})$ satisfies

$$
\widetilde{\mathcal{S}}_{s}^{\left[k_{s}\right]}(t) \cdots \widetilde{\mathcal{S}}_{1}^{\left[k_{1}\right]}(t)-\mathcal{E}_{H}(t) \mathcal{S}_{s}^{\left[k_{s}\right]}(0) \cdots \mathcal{S}_{1}^{\left[k_{1}\right]}(0)=\mathscr{O}(t),
$$

and summing up we obtain

$$
\widetilde{\mathcal{S}}^{[p+1]}(t)-\mathcal{E}_{H}(t) \mathcal{S}^{[p+1]}(0)=\mathscr{O}(t) .
$$

(iii) Splitting the term (6.5) to be estimated into

$\mathcal{S}^{[p+1]}(t)-\mathcal{E}_{H}(t) \mathcal{S}^{[p+1]}(0)=\left(\mathcal{S}^{[p+1]}(t)-\widetilde{\mathcal{S}}^{[p+1]}(t)\right)+\left(\widetilde{\mathcal{S}}^{[p+1]}(t)-\mathcal{E}_{H}(t) \mathcal{S}^{[p+1]}(0)\right)$,

it remains to study

$$
\mathcal{S}^{[p+1]}-\widetilde{\mathcal{S}}^{[p+1]}=\sum_{k \in \mathbb{N}_{0}^{s},|k|=p+1}\left(\begin{array}{c}
p+1 \\
k
\end{array}\right)\left(\mathcal{S}_{s}^{\left[k_{s}\right]} \ldots \mathcal{S}_{1}^{\left[k_{1}\right]}-\widetilde{\mathcal{S}}_{s}^{\left[k_{s}\right]} \ldots \widetilde{\mathcal{S}}_{1}^{\left[k_{1}\right]}\right) .
$$

The terms (differences of products) under the sum can be written as

$$
\begin{aligned}
\mathcal{S}_{s}^{\left[k_{s}\right]} \ldots \mathcal{S}_{1}^{\left[k_{1}\right]}-\widetilde{\mathcal{S}}_{s}^{\left[k_{s}\right]} \ldots \widetilde{\mathcal{S}}_{1}^{\left[k_{1}\right]}= & \left(\mathcal{S}_{s}^{\left[k_{s}\right]}-\widetilde{\mathcal{S}}_{s}^{\left[k_{s}\right]}\right) \mathcal{S}_{s-1}^{\left[k_{s-1}\right]} \ldots \mathcal{S}_{1}^{\left[k_{1}\right]} \\
& +\widetilde{\mathcal{S}}_{s}^{\left[k_{s}\right]}\left(\mathcal{S}_{s-1}^{\left[k_{s-1}\right]}-\widetilde{\mathcal{S}}_{s-1}^{\left[k_{s-1}\right]}\right) \mathcal{S}_{s-2}^{\left[k_{s-2}\right]} \ldots \mathcal{S}_{1}^{\left[k_{1}\right]} \\
& +\ldots \\
& +\widetilde{\mathcal{S}}_{s}^{\left[k_{s}\right]} \ldots \widetilde{\mathcal{S}}_{1}^{\left[k_{1}\right]}\left(\mathcal{S}_{1}^{\left[k_{1}\right]}-\widetilde{\mathcal{S}}_{1}^{\left[k_{1}\right]}\right) .
\end{aligned}
$$

From Lemma 2,

$$
\mathcal{S}_{j}^{[k]}=\sum_{\ell=0}^{k}\left(\begin{array}{l}
k \\
\ell
\end{array}\right) \mathcal{W}_{j}^{[k-\ell]} \mathcal{V}_{j}^{[\ell]}=\mathscr{O}(1)
$$

and from $(6.7 \mathrm{~b})$,

$$
\widetilde{\mathcal{S}}_{j}^{[k]}=\sum_{\ell=0}^{k}\left(\begin{array}{l}
k \\
\ell
\end{array}\right) \widetilde{\mathcal{W}}_{j}^{[k-\ell]} \widetilde{\mathcal{V}}_{j}^{[\ell]}=\mathscr{O}(1)
$$


Furthermore,

$$
\mathcal{S}_{j}^{[k]}-\widetilde{\mathcal{S}}_{j}^{[k]}=\sum_{\ell=0}^{k}\left(\begin{array}{l}
k \\
\ell
\end{array}\right)\left(\mathcal{W}_{j}^{[k-\ell]} \mathcal{V}_{j}^{[\ell]}-\widetilde{\mathcal{W}}_{j}^{[k-\ell]} \widetilde{\mathcal{V}}_{j}^{[\ell]}\right),
$$

with

$$
\begin{aligned}
& \mathcal{W}_{j}^{[k-\ell]} \mathcal{V}_{j}^{[\ell]}-\widetilde{\mathcal{W}}_{j}^{[k-\ell]} \widetilde{\mathcal{V}}_{j}^{[\ell]} \\
& =\mathcal{W}_{j}^{[k-\ell]}\left(\mathcal{V}_{j}^{[\ell]}-\widetilde{\mathcal{V}}_{j}^{[\ell]}\right)+\left(\mathcal{W}_{j}^{[k-\ell]}-\widetilde{\mathcal{W}}_{j}^{[k-\ell]}\right) \widetilde{\mathcal{V}}_{j}^{[\ell]}=\mathscr{O}(t)
\end{aligned}
$$

because

$$
\mathcal{V}_{j}^{[\ell]}(t)-\widetilde{\mathcal{V}}_{j}^{[\ell]}(t)=\int_{0}^{t} \mathcal{E}_{A_{j}}(t-\tau) \rho_{j}\left(\mathcal{V}_{j}^{[k]}\right)(\tau) \mathrm{d} \tau=\mathscr{O}(t)
$$

$($ see $(5.9 \mathrm{c}),(6.7 \mathrm{c}))$, and

$$
\mathcal{W}_{j}^{[k-\ell]}(t)-\widetilde{\mathcal{W}}_{j}^{[k-\ell]}(t)=\int_{0}^{t} \mathcal{E}_{C_{j}}(t-\tau) \sigma_{j}\left(\mathcal{W}_{j}^{[k]}\right)(\tau) \mathcal{E}_{B_{j}}(t-\tau) \mathrm{d} \tau=\mathscr{O}(t)
$$

(see $(5.10 \mathrm{c}),(6.7 \mathrm{c}))$

From these preliminary considerations we obtain

$$
\mathcal{S}^{[p+1]}(t)-\widetilde{\mathcal{S}}^{[p+1]}(t)=\mathscr{O}(t) .
$$

Altogether, (6.9a) and (6.9b) imply (6.5). Thus we have established the following result:

\section{Proposition 2}

$$
\mathcal{P}(t) u-\mathcal{L}(t) u=\mathscr{O}\left(t^{p+2}\right),
$$

that is, the local error estimate (6.1b) is asymptotically correct.

\section{Practical realization, extension to nonlinear equations}

As before, it is convenient to consider the time interval $[0, t]$ with stepsize $t>0$. The extension to an arbitrary integration step $t \mapsto t+\Delta t$ with stepsize $\Delta t>0$ is straightforward.

7.1 Practical evaluation of the defect

For the practical implementation of our error estimators, the defect can be evaluated by a Horner-type scheme, which we illustrate for the case $s=3$, using the notation from Section 5 and recalling the definition of $\mathcal{V}_{j}, \mathcal{W}_{j}$, see (2.2):

$$
\begin{aligned}
\mathcal{D}:= & \mathcal{S}^{[1]}=\frac{\mathrm{d}}{\mathrm{d} t}\left(\mathcal{S}_{3} \mathcal{S}_{2} \mathcal{S}_{1}\right)-H \mathcal{S}_{3} \mathcal{S}_{2} \mathcal{S}_{1} \\
= & \frac{\mathrm{d}}{\mathrm{d} t} \mathcal{S}_{3} \cdot \mathcal{S}_{2} \cdot \mathcal{S}_{1}+\mathcal{S}_{3} \cdot \frac{\mathrm{d}}{\mathrm{d} t} \mathcal{S}_{2} \cdot \mathcal{S}_{1}+\mathcal{S}_{3} \cdot \mathcal{S}_{2} \cdot \frac{\mathrm{d}}{\mathrm{d} t} \mathcal{S}_{1}-H \mathcal{S}_{3} \mathcal{S}_{2} \mathcal{S}_{1} \\
= & \left(\mathcal{W}_{3} D_{3} \mathcal{V}_{3}+C_{3} \mathcal{S}_{3}\right) \mathcal{S}_{2} \mathcal{S}_{1} \\
& +\mathcal{W}_{3} \mathcal{V}_{3}\left(\left(\mathcal{W}_{2} D_{2} \mathcal{V}_{2}+C_{2} \mathcal{S}_{2}\right) \mathcal{S}_{1}+\mathcal{W}_{2} \mathcal{V}_{2}\left(\mathcal{W}_{1} D_{1} \mathcal{V}_{1}+C_{1} \mathcal{S}_{1}\right)\right) \\
& -\left(H-C_{3}+C_{3} \mathcal{S}_{3} \mathcal{S}_{2} \mathcal{S}_{1}\right.
\end{aligned}
$$


resulting in

$$
\begin{aligned}
\mathcal{D}= & \mathcal{W}_{3}\left\{D_{3} \mathcal{V}_{3} \mathcal{S}_{2} \mathcal{S}_{1}+\mathcal{V}_{3}\left\{C_{2} \mathcal{S}_{2} \mathcal{S}_{1}+\mathcal{W}_{2}\left\{D_{2} \mathcal{V}_{2} \mathcal{S}_{1}+\mathcal{V}_{2}\left\{C_{1} \mathcal{S}_{1}+\mathcal{W}_{1} D_{1} \mathcal{V}_{1}\right\}\right\}\right\}\right\} \\
& -\left(H-C_{3}\right) \mathcal{S}_{3} \mathcal{S}_{2} \mathcal{S}_{1}
\end{aligned}
$$

Computationally it is advantageous to evaluate (7.1) in parallel to the evaluation of $\mathcal{S}=\mathcal{S}_{3} \mathcal{S}_{2} \mathcal{S}_{1}$. A closer investigation shows that for such an evaluation of $\mathcal{S} u$ and $\mathcal{D} u$, storage for 5 vectors is required (where each intermediate result which is not required further is overwritten). The pattern is similar as for the AB case. The computational cost is thus the following:

$-\mathcal{S} u$ requires evaluation of $3 s$ exponentials.

$-\mathcal{D} u$ requires evaluation of $\approx 3 s$ exponentials and $\approx 3 s$ operators.

For practical schemes, in particular in the symmetric case, the number of evaluations is significantly lower due to a number of vanishing coefficients.

\subsection{The nonlinear case}

Consider a nonlinear evolution equation

$$
\left\{\begin{array}{l}
u^{\prime}=H(u)=A(u)+B(u)+C(u), \quad t \geq 0, \\
\mathbf{u}(0) \text { given, }
\end{array}\right.
$$

and corresponding (sub-)flows $\mathcal{E}_{H}(t, u)$ and $\mathcal{E}_{A}(t, u), \mathcal{E}_{B}(t, u), \mathcal{E}_{C}(t, u)$. For the case $s=3$, for instance, a step of an $s$-stage splitting method takes the form

$$
\begin{aligned}
& u_{1}=\mathcal{E}_{A}\left(a_{1} t, u\right), \quad v_{1}=\mathcal{E}_{B}\left(b_{1} t, u_{1}\right), \quad w_{1}=\mathcal{E}_{C}\left(c_{1} t, v_{1}\right)=\mathcal{S}_{1}(t, u), \\
& u_{2}=\mathcal{E}_{A}\left(a_{2} t, w_{1}\right), v_{2}=\mathcal{E}_{B}\left(b_{2} t, u_{2}\right), w_{2}=\mathcal{E}_{C}\left(c_{2} t, v_{2}\right)=\mathcal{S}_{2}\left(t, w_{1}\right) \text {, } \\
& u_{3}=\mathcal{E}_{A}\left(a_{3} t, w_{2}\right), v_{3}=\mathcal{E}_{B}\left(b_{3} t, u_{3}\right), w_{3}=\mathcal{E}_{C}\left(c_{3} t, v_{3}\right)=\mathcal{S}_{3}\left(t, w_{2}\right) \text {, } \\
& \mathcal{S}(t, u)=w_{3}=\mathcal{S}_{3}\left(t, \mathcal{S}_{2}\left(t, \mathcal{S}_{1}(t, u)\right)\right),
\end{aligned}
$$

with local error

$$
\mathcal{L}(t, u)=\mathcal{S}(t, u)-\mathcal{E}_{H}(t, u)
$$

The nonlinear analogue of the local error respresentation (2.12) is obtained via the nonlinear variation-of-constants formula

$$
\mathcal{L}(t, u)=\int_{0}^{t} \partial_{2} \mathcal{E}_{H}(t-\tau, \mathcal{S}(\tau, u)) \cdot \mathcal{D}(\tau, u) \mathrm{d} \tau,
$$

with the defect

$$
\mathcal{D}(t, u)=\frac{\mathrm{d}}{\mathrm{d} t} \mathcal{S}(t, u)-H(\mathcal{S}(t, u)) .
$$

A defect-based local error estimate is defined in a way completely analogous to $(6.1 \mathrm{~b})$,

$$
\mathcal{P}(t) u=\frac{t}{p+1} \mathcal{D}(t) u
$$

For a detailed analysis for the nonlinear case in a simpler setting (splitting into two operators and low order methods) we refer to [11]. Here we illustrate the algorithmic evaluation of $\mathcal{D}(t, u)$ for the case $s=3$, with obvious generalization to general $s$-stage schemes. To this end, as for the linear case, we rewrite the defect in a way such no 
derivatives w.r.t. $t$ appear. However, evaluation of the Fréchet derivatives of nonlinear subflows with respect to initial values are required.

With $u_{j}=u_{j}(t), v_{j}=v_{j}(t), w_{j}=w_{j}(t)$ we have

$$
\begin{aligned}
\frac{\mathrm{d}}{\mathrm{d} t} \mathcal{S}(t, u)=\frac{\mathrm{d}}{\mathrm{d} t} w_{3}(t) & =\frac{\mathrm{d}}{\mathrm{d} t} \mathcal{E}_{C}\left(c_{3} t, v_{3}(t)\right) \\
& =\frac{\partial}{\partial t} \mathcal{E}_{C}\left(c_{3} t, v_{3}(t)\right)+\partial_{2} \mathcal{E}_{C}\left(c_{3} t, v_{3}(t)\right) \cdot \frac{\mathrm{d}}{\mathrm{d} t} v_{3}(t) \\
& =c_{3} C\left(w_{3}(t)\right)+\partial_{2} \mathcal{E}_{C}\left(c_{3} t, v_{3}(t)\right) \cdot \frac{\mathrm{d}}{\mathrm{d} t} v_{3}(t) .
\end{aligned}
$$

We recall the fundamental identity ${ }^{6}[11]$

$$
\partial_{2} \mathcal{E}_{F}(t, v) \cdot F(v)=F\left(\mathcal{E}_{F}(t, v)\right) .
$$

Resorting to (7.6) for $F=B$, we obtain

$$
\begin{aligned}
\frac{\mathrm{d}}{\mathrm{d} t} v_{3}(t) & =\frac{\mathrm{d}}{\mathrm{d} t} \mathcal{E}_{B}\left(b_{3} t, u_{3}(t)\right) \\
& =\frac{\partial}{\partial t} \mathcal{E}_{B}\left(b_{3} t, u_{3}(t)\right)+\partial_{2} \mathcal{E}_{B}\left(b_{3} u_{3}(t)\right) \cdot \frac{\mathrm{d}}{\mathrm{d} t} u_{3}(t) \\
& =b_{3} B\left(u_{3}(t)\right)+\partial_{2} \mathcal{E}_{B}\left(b_{3} t, u_{3}(t)\right) \cdot \frac{\mathrm{d}}{\mathrm{d} t} u_{3}(t) \\
& =b_{3} \partial_{2} \mathcal{E}_{B}\left(b_{3} t, u_{3}(t)\right) \cdot B\left(u_{3}(t)\right)+\partial_{2} \mathcal{E}_{B}\left(b_{3} t, u_{3}(t)\right) \cdot \frac{\mathrm{d}}{\mathrm{d} t} u_{3}(t) \\
& =\partial_{2} \mathcal{E}_{B}\left(b_{3} t, u_{3}(t)\right) \cdot\left\{b_{3} B\left(u_{3}(t)\right)+\frac{\mathrm{d}}{\mathrm{d} t} u_{3}(t)\right\},
\end{aligned}
$$

and similarly for $F=A$,

$$
\begin{aligned}
\frac{\mathrm{d}}{\mathrm{d} t} u_{3}(t) & =\frac{\mathrm{d}}{\mathrm{d} t} \mathcal{E}_{A}\left(a_{3} t, w_{2}(t)\right) \\
& =\frac{\partial}{\partial t} \mathcal{E}_{A}\left(a_{3} t, w_{2}(t)\right)+\partial_{2} \mathcal{E}_{A}\left(a_{3} w_{2}(t)\right) \cdot \frac{\mathrm{d}}{\mathrm{d} t} w_{2}(t) \\
& =a_{3} A\left(u_{3}(t)\right)+\partial_{2} \mathcal{E}_{A}\left(a_{3} t, w_{2}(t)\right) \cdot \frac{\mathrm{d}}{\mathrm{d} t} w_{2}(t) \\
& =a_{3} \partial_{2} \mathcal{E}_{A}\left(a_{3} t, w_{2}(t)\right) \cdot A\left(w_{2}(t)\right)+\partial_{2} \mathcal{E}_{A}\left(a_{3} t, w_{2}(t)\right) \cdot \frac{\mathrm{d}}{\mathrm{d} t} w_{2}(t) \\
& =\partial_{2} \mathcal{E}_{A}\left(a_{3} t, w_{2}(t)\right) \cdot\left\{a_{3} A\left(w_{2}(t)\right)+\frac{\mathrm{d}}{\mathrm{d} t} w_{2}(t)\right\} .
\end{aligned}
$$

This implies

$$
\begin{aligned}
\frac{\mathrm{d}}{\mathrm{d} t} \mathcal{S}(t, u)= & c{ }_{3} C\left(w_{3}(t)\right) \\
+ & \partial_{2} \mathcal{E}_{C}\left(c_{3} t, v_{3}(t)\right) \cdot \partial_{2} \mathcal{E}_{B}\left(b_{3} t, u_{3}(t)\right) \cdot \\
& \cdot\left\{b_{3} B\left(u_{3}(t)\right)+\partial_{2} \mathcal{E}_{A}\left(a_{3} t, w_{2}(t)\right) \cdot\left\{a_{3} A\left(w_{2}(t)\right)+\frac{\mathrm{d}}{\mathrm{d} t} w_{2}(t)\right\}\right\} .
\end{aligned}
$$

Systematically continuing this expansion leads to a Horner-type representation of $\frac{\mathrm{d}}{\mathrm{d} t} \mathcal{S}(t, u)$, which eventually results into

$$
\begin{aligned}
\mathcal{D}(t, u)=\partial_{2} \mathcal{E}_{C}\left(c_{3} t, v_{3}\right) \cdot \partial_{2} \mathcal{E}_{B}\left(b_{3} t, u_{3}\right) \cdot & \cdot\left\{b_{3} B\left(u_{3}\right)+\partial_{2} \mathcal{E}_{A}\left(a_{3} t, w_{2}\right) \cdot\right. \\
& \cdot\left\{a_{3} A\left(w_{2}\right)+c_{2} C\left(w_{2}\right)+\right. \\
+ & \partial_{2} \mathcal{E}_{C}\left(c_{2} t, v_{2}\right) \cdot \partial_{2} \mathcal{E}_{B}\left(b_{2} t, u_{2}\right) \cdot \\
\cdot & \left\{b_{2} B\left(u_{2}\right)+\partial_{2} \mathcal{E}_{A}\left(a_{2} t, w_{1}\right) \cdot\right. \\
& \cdot\left\{a_{2} A\left(w_{1}\right)+c_{1} C\left(w_{1}\right)+\right. \\
+ & \partial_{2} \mathcal{E}_{C}\left(c_{1} t, v_{1}\right) \cdot \partial_{2} \mathcal{E}_{B}\left(b_{1} t, u_{1}\right) \cdot \\
& \left.\left.\left.\left.\cdot\left\{b_{1} B\left(u_{1}\right)+\partial_{2} \mathcal{E}_{A}\left(a_{1} t, u\right) \cdot a_{1} A(u)\right\}\right\}\right\}\right\}\right\} \\
-A\left(w_{3}\right)- & B\left(w_{3}\right)-\left(1-c_{3}\right) C\left(w_{3}\right),
\end{aligned}
$$

\footnotetext{
${ }^{6}$ For a linear operator $F,(7.6)$ reduces to the identity $\mathcal{E}_{F}(t) \cdot F \cdot v=F \cdot \mathcal{E}_{F}(t) \cdot v$, with $\mathcal{E}_{F}(t)=\mathrm{e}^{t F}$
} 
which is the nonlinear version of (7.1). For details on the concrete computational realization, we refer to the numerical examples given next.

\section{Numerical examples}

In the following, we confirm asymptotical correctness of our a posteriori local error estimators based on (7.5) associated with different splitting methods of when applied to nonlinear evolution equations. We consider test problems of Schrödinger and of parabolic type, where splitting into three operators permits a considerable reduction in the complexity of the subproblems to be solved, which results from freezing either the time variable or a solution component. Evaluation of the defect $\mathcal{D}(t, u)$ is performed according to (7.7). Furthermore, for non-autonomous problems as (8.1), employing a standard reformulation as an autonomous system allows to apply the general framework. We note that qualitatively the same numerical results are obtained for appropriately simplified linear test problems.

Schrödinger equation We consider the Gross-Pitaevskii equation [16,17] in one space dimension under a harmonic potential with $\vartheta=1$,

$$
\begin{aligned}
\mathrm{i} \partial_{t} \psi(x, t)=- & \frac{1}{2} \partial_{x x} \psi(x, t)+\frac{1}{2} x^{2} \psi(x, t) \\
& +\vartheta|\psi(x, t)|^{2} \psi(x, t)+r(x, t), \quad(x, t) \in(-8,8) \times(0,1) ;
\end{aligned}
$$

the additional inhomogeneity $r$ is chosen such that the true solution is given by the ground state solution of the linear Schrödinger equation $(\vartheta=0)$. The structure of this partial differential equation suggests a splitting into three parts. In view of (7.7), we specify the subproblems and indicate the computation of the corresponding Fréchet derivatives of the evolution operators with respect to initial values.

Part A. The first part is given by the Laplace operator. Due to the linearity of the subproblem, the evolution operator $\mathcal{E}_{A}$ and the Fréchet derivative $\partial_{2} \mathcal{E}_{A}$ coincide.

Part B. The second part is defined by potential and the nonlinearity. The exact solution to this subproblem is given by

$$
\left(\mathcal{E}_{B}(t, u)\right)(x)=\mathrm{e}^{-\mathrm{i} t\left(\frac{1}{2} x^{2}+\vartheta|u(x)|^{2}\right)} u(x)
$$

which also allows a straightforward computation of the Fréchet derivative $\partial_{2} \mathcal{E}_{B}$.

Part C. The third part involves the inhomogeneity $r(x, t)$. In order to cast this subproblem into our general framework, we use a standard reformulation as autonomous system

$$
\partial_{t}\left(\begin{array}{c}
\psi(x, t) \\
t
\end{array}\right)=\left(\begin{array}{c}
r(x, t) \\
1
\end{array}\right)
$$

In this example, the integration can be performed exactly. For each substep of length $\Delta t$, starting at time $t$, evalutation of the associated Fréchet derivative $\partial_{2} \mathcal{E}_{C}$ amounts to multiplication by

$$
\left(\begin{array}{cc}
1 & r(x, t+\Delta t)-r(x, t) \\
0 & 1
\end{array}\right) .
$$


For space discretization, we use Fast Fourier techniques with $M=100$ grid points. For time discretization, we apply the first-order Lie-Trotter splitting method $(p=1)$, the second-order Strang splitting method $(p=2)$, a three-stage third-order splitting method involving real coefficients $(p=3)$, and a fourth-order splitting method $(p=4)$ obtained by composition according to Yoshida [14].

Diffusion-reaction system We study the Gray-Scott equations $[18,19]$ in a single space dimension

$$
\begin{gathered}
\left\{\begin{array}{l}
\partial_{t} u(x, t)=\left(d_{u} \partial_{x x}-c_{u}\right) u(x, t)+c_{u}-u(x, t)(v(x, t))^{2}, \\
\partial_{t} v(x, t)=\left(d_{v} \partial_{x x}-c_{v}\right) v(x, t)+u(x, t)(v(x, t))^{2},
\end{array}\right. \\
d_{u}=0.001, \quad d_{v}=0.0001, \quad c_{u}=0.04, \quad c_{v}=0.1, \\
u(x, 0)=\mathrm{e}^{-2 x^{2}}, \quad v(x, 0)=0.1+\mathrm{e}^{-4 x^{2}}, \quad(x, t) \in(-1.5 \pi, 1.5 \pi) \times(0,1),
\end{gathered}
$$

subject to periodic boundary conditions. We make use of the fact that a splitting of the nonlinear part, acting pointwise in the spatial variable, into a pair of simple nonlinear ordinary differential equations

$$
\left\{\begin{array} { l } 
{ \partial _ { t } u ( x , t ) = - u ( x , t ) ( v ( x , t ) ) ^ { 2 } , } \\
{ \partial _ { t } v ( x , t ) = 0 , }
\end{array} \quad \left\{\begin{array}{l}
\partial_{t} u(x, t)=0 \\
\partial_{t} v(x, t)=u(x, t)(v(x, t))^{2}
\end{array}\right.\right.
$$

permits to determine the exact solutions to these subproblems as well as their Fréchet derivatives with respect to initial values in an easy manner. For space discretization, we use a Fourier spectral method at $M=512$ grid points. For time discretization, we apply the first-order Lie-Trotter splitting method $(p=1)$, the second-order Strang splitting method $(p=2)$, a four-stage third-order splitting methods involving complex coefficients $(p=3)$, and a fourth-order splitting method involving complex coefficients, obtained in analogy to Yoshida's scheme $(p=4)$.

In Figures 1 and 2 the global errors versus the constant time stepsizes are displayed. In addition, the global errors of the numerical approximations obtained through a correction of $\mathcal{S}$ according to

$$
\mathcal{S}-\mathcal{P}
$$

in each time step are included; as usual, $\mathcal{S}$ denotes the splitting approximation of order $p$ and $\mathcal{P}$ the a posteriori local error estimator. The numerical results show that the improved approximation is of global order $p+1$, which confirms asymptotical correctness of the a posteriori local error estimator. 

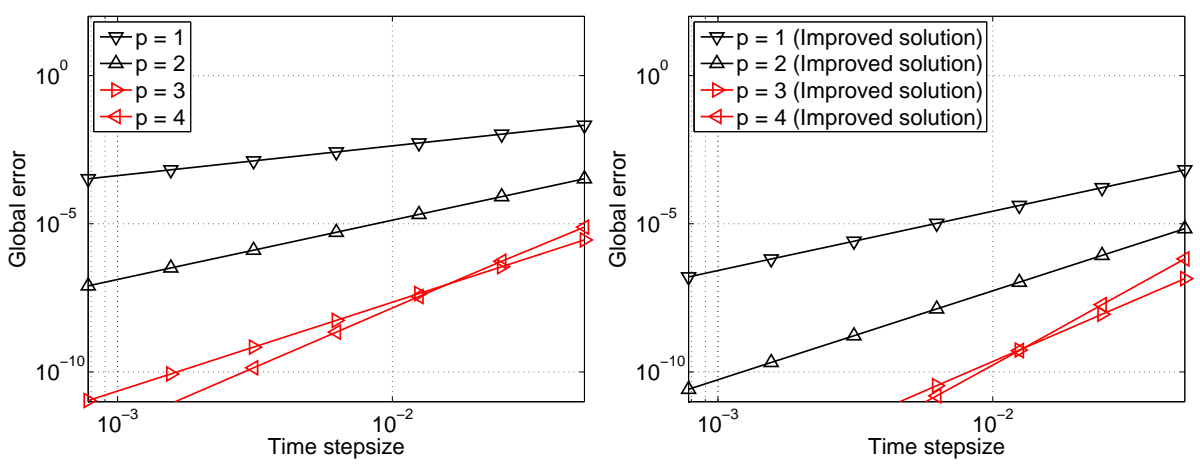

Fig. 1 Time integration of Gross-Pitaevskii equation (8.1). Global errors versus time stepsizes of different splitting methods (left) and associated corrected approximations (right).
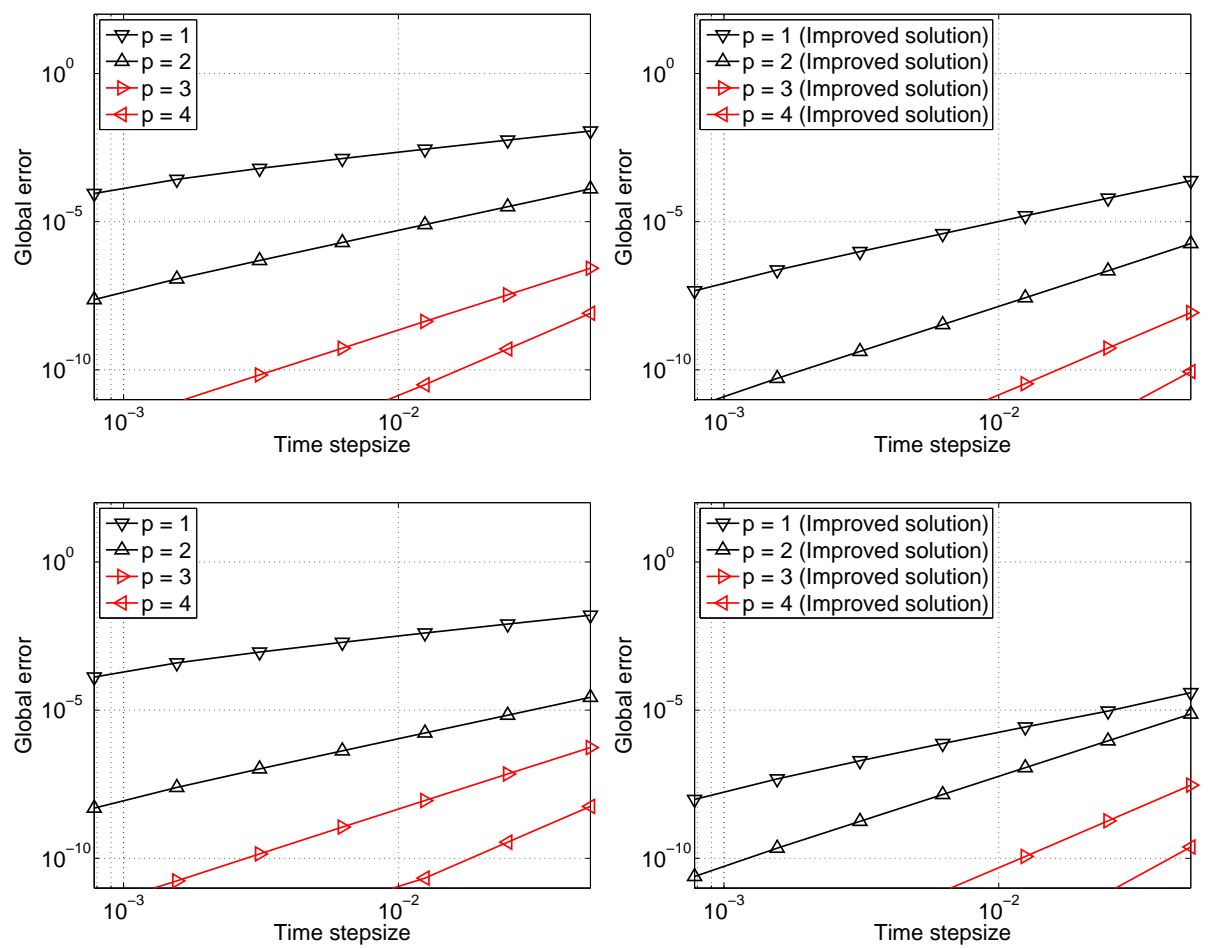

Fig. 2 Time integration of Gray-Scott equations (8.5). Global errors versus time stepsizes of different splitting methods (left) and associated corrected approximations (right). First (first row) and second (second row) component of the solution. 


\section{A Auxiliary identities and results}

The commutator

$$
[X, Y]=X Y-Y X
$$

satisfies the following fundamental identities:

$$
\begin{aligned}
{[X Y, Z] } & =X[Y, Z]+[X, Z] Y, \\
0 & =[[X, Y], Z]+[[Y, Z], X]+[[Z, X], Y] \quad \text { (Jacobi identity). }
\end{aligned}
$$

Lemma 4 Let $A, K$ denote operators not depending on $t$ and $\mathcal{G}=\mathcal{G}(t)$ a time-dependent inhomogeneity. Assume that $\mathcal{X}$ is the solution of the initial value problem

$$
\left\{\begin{array}{l}
\frac{\mathrm{d}}{\mathrm{d} t} \mathcal{X}=A \mathcal{X}+\mathcal{G} \\
\mathcal{X}(0) \text { given }
\end{array}\right.
$$

Then the commutator $\mathcal{Y}:=[\mathcal{X}, K]$ satisfies the initial value problem

$$
\left\{\begin{array}{l}
\frac{\mathrm{d}}{\mathrm{d} t} \mathcal{Y}=A \mathcal{Y}+[A, K] \mathcal{X}+[\mathcal{G}, K] \\
\mathcal{Y}(0)=[\mathcal{X}(0), K]
\end{array}\right.
$$

Proof We differentiate using identity (A.2a):

$$
\begin{aligned}
\frac{\mathrm{d}}{\mathrm{d} t}[\mathcal{X}, K]=\left[\frac{\mathrm{d}}{\mathrm{d} t} \mathcal{X}, K\right] & =[A \mathcal{X}, K]+[\mathcal{G}, K] \\
& =A[\mathcal{X}, K]+[A, K] \mathcal{X}+[\mathcal{G}, K],
\end{aligned}
$$

which completes the proof.

Lemma 5 [13, Lemma 1]. Let $B, C, K$ denote operators not depending on $t$ and $\mathcal{G}=\mathcal{G}(t)$ a time-dependent inhomogeneity. Consider the inhomogeneous Sylvester equation

$$
\left\{\begin{array}{l}
\frac{\mathrm{d}}{\mathrm{d} t} \mathcal{X}=\mathcal{X} B+C \mathcal{X}+\mathcal{G} \\
\mathcal{X}(0) \text { given }
\end{array}\right.
$$

(i) The initial value problem (A.5) admits the solution representation

$$
\mathcal{X}(t)=\mathcal{E}_{C}(t) \mathcal{X}(0) \mathcal{E}_{B}(t)+\int_{0}^{t} \mathcal{E}_{C}(t-\tau) \mathcal{G}(\tau) \mathcal{E}_{B}(t-\tau) \mathrm{d} \tau
$$

(ii) Provided that $\mathcal{X}$ satisfies the Sylvester equation (A.5), the time-dependent operators $\mathcal{Y}$ and $\mathcal{Z}$, defined by

$$
\mathcal{Y}(t)=\mathcal{X}(t) K, \quad \mathcal{Z}(t)=K \mathcal{X}(t),
$$

are solutions to the Sylvester equations

$$
\left\{\begin{array}{l}
\frac{\mathrm{d}}{\mathrm{d} t} \mathcal{Y}=\mathcal{Y} B+C \mathcal{Y}+\mathcal{X}[B, K]+\mathcal{G} K \\
\mathcal{Y}(0)=\mathcal{X}(0) K
\end{array}\right.
$$

and

$$
\left\{\begin{array}{l}
\frac{\mathrm{d}}{\mathrm{d} t} \mathcal{Z}=\mathcal{Z} B+C \mathcal{Z}+[K, C] \mathcal{X}+K \mathcal{G} \\
\mathcal{Z}(0)=K \mathcal{X}(0)
\end{array}\right.
$$

respectively.

(iii) Provided that $\mathcal{X}$ satisfies the Sylvester equation $(\mathrm{A} .5)$, the commutator $\mathcal{Y}=[\mathcal{X}, K]$ is the solution of the Sylvester equation

$$
\left\{\begin{array}{l}
\frac{\mathrm{d}}{\mathrm{d} t} \mathcal{Y}=\mathcal{Y} B+C \mathcal{Y}+\mathcal{X}[B, K]+[C, K] \mathcal{X}+[\mathcal{G}, K] \\
\mathcal{Y}(0)=[\mathcal{X}(0), K]
\end{array}\right.
$$

Proof See [13]. 


\section{B Proofs of Lemmas 2 and 3}

In the following, we prove statements about the quantities $H_{j}, \mathcal{S}_{j}^{[k]} \ldots$, etc. However, for convenience of notation we suppress the index $j$. In particular, $\delta(\cdot), \rho(\cdot), \sigma(\cdot)$ are to be identified with $\delta_{j}(\cdot), \rho_{j}(\cdot), \sigma_{j}(\cdot)$ defined in $(2.4)$.

With (cf. (2.4b), (2.4c))

$$
\begin{aligned}
\rho(\mathcal{X}) & =\frac{\mathrm{d}}{\mathrm{d} t} \mathcal{X}-A \mathcal{X}, \\
\sigma(\mathcal{X}) & =\frac{\mathrm{d}}{\mathrm{d} t} \mathcal{X}-\mathcal{X} B-C \mathcal{X},
\end{aligned}
$$

the identities (A.4) and (A.8) take the form

$$
\begin{aligned}
\rho([\mathcal{X}, K]) & =[A, K] \mathcal{X}+[\rho(\mathcal{X}), K] \\
\sigma([\mathcal{X}, K]) & =\mathcal{X}[B, K]+[C, K] \mathcal{X}+[\sigma(\mathcal{X}), K] .
\end{aligned}
$$

We will make repeated use of these identities in the proof of Lemma 3 below. Furthermore, (A.2a) is used several times.

Proof of Lemma 2. For $k=0$, identity (5.8) is the same as $\mathcal{S}^{[0]}=\mathcal{W}^{[0]} \mathcal{V}^{[0]}$. For the general induction step $k \mapsto k+1$, by (5.5) and the inductive assumption (5.8) we have

$$
\begin{aligned}
\mathcal{S}^{[k+1]} & =\left[\mathcal{S}^{[k]}, \underline{H}\right]+\delta\left(\mathcal{S}^{[k]}\right) \\
& =\sum_{\ell=0}^{k}\left(\begin{array}{l}
k \\
\ell
\end{array}\right)\left(\left[\mathcal{W}^{[k-\ell]} \mathcal{V}^{[\ell]}, \underline{H}\right]+\delta\left(\mathcal{W}^{[k-\ell]} \mathcal{V}^{[\ell]}\right)\right),
\end{aligned}
$$

with (using (A.2a))

$$
\begin{aligned}
{[} & \left.\mathcal{W}^{[k-\ell]} \mathcal{V}^{[\ell]}, \underline{H}\right]+\delta\left(\mathcal{W}^{[k-\ell]} \mathcal{V}^{[\ell]}\right) \\
= & \mathcal{W}^{[k-\ell]}\left[\mathcal{V}^{[\ell]}, \underline{H}\right]+\left[\mathcal{W}^{[k-\ell]}, \underline{H}\right] \mathcal{V}^{[\ell]} \\
& +\frac{\mathrm{d}}{\mathrm{d} t}\left(\mathcal{W}^{[k-\ell]} \mathcal{V}^{[\ell]}\right)-H \mathcal{W}^{[k-\ell]} \mathcal{V}^{[\ell]} \\
= & \mathcal{W}^{[k-\ell]}\left[\mathcal{V}^{[\ell]}, \underline{H}\right]+\left[\mathcal{W}^{[k-\ell]}, \underline{H}\right] \mathcal{V}^{[\ell]} \\
& +\frac{\mathrm{d}}{\mathrm{d} t} \mathcal{W}^{[k-\ell]} \cdot \mathcal{V}^{[\ell]}+\mathcal{W}^{[k-\ell]} \cdot \frac{\mathrm{d}}{\mathrm{d} t} \mathcal{V}^{[\ell]}-H \mathcal{W}^{[k-\ell]} \mathcal{V}^{[\ell]} \\
= & \mathcal{W}^{[k-\ell]}\left[\mathcal{V}^{[\ell]}, \underline{H}\right]+\left[\mathcal{W}^{[k-\ell]}, \underline{H}\right] \mathcal{V}^{[\ell]} \\
& +\left(\mathcal{W}^{[k-\ell]} B+C \mathcal{W}^{[k-\ell]}+\sigma\left(\mathcal{W}^{[k-\ell]}\right)\right) \mathcal{V}^{[\ell]} \\
& +\mathcal{W}^{[k-\ell]}\left(A \mathcal{V} \mathcal{V}^{[\ell]}+\rho\left(\mathcal{V}^{[\ell]}\right)\right)-H \mathcal{W}^{[k-\ell]} \mathcal{V}^{[\ell]} \\
= & \mathcal{W}^{[k-\ell]}\left(\left[\mathcal{V}^{[\ell]}, \underline{H}\right]+\rho\left(\mathcal{V}^{[\ell]}\right)\right)+\left(\left[\mathcal{W}^{[k-\ell]}, \underline{H}\right]+\sigma\left(\mathcal{W}^{[k-\ell]}\right)\right) \mathcal{V}^{[\ell]} \\
& +\mathcal{W}^{[k-\ell]} D \mathcal{V}^{[\ell]}+C \mathcal{W}^{[k-\ell]} \mathcal{V}^{[\ell]}-H \mathcal{W}^{[k-\ell]} \mathcal{V}^{[\ell]} \\
= & \mathcal{W}^{[k-\ell]}\left(\left[\mathcal{V}^{[\ell]}, \underline{H}\right]+\rho\left(\mathcal{V}^{[\ell]}\right)\right)+\left(\left[\mathcal{W}^{[k-\ell]}, \underline{H}+D\right]+\sigma\left(\mathcal{W}^{[k-\ell]}\right)\right) \mathcal{V}^{[\ell]} \\
& +\underbrace{[k-\ell]}_{=0} D \mathcal{V}^{[\ell]}+C \mathcal{W}^{[k-\ell]} \mathcal{V}^{[\ell]}-\mathcal{W}^{[k-\ell]} D \mathcal{V}^{[\ell]}+D \mathcal{W}^{[k-\ell]} \mathcal{V}^{[\ell]}-H \mathcal{W}^{[k-\ell]} \mathcal{V}^{[\ell]} \\
= & \mathcal{W}^{[k-\ell]} \mathcal{V}^{[\ell+1]}+\mathcal{W}^{[k+1-\ell]} \mathcal{V}^{[\ell]},
\end{aligned}
$$

by definition of $\mathcal{V}^{[\ell+1]}$ and $\mathcal{W}^{[k+1-\ell]}$. Altogether,

$$
\begin{aligned}
\mathcal{S}^{[k+1]} & =\sum_{\ell=0}^{k}\left(\begin{array}{c}
k \\
\ell
\end{array}\right) \mathcal{W}^{[k-\ell]} \mathcal{V}^{[\ell+1]}+\sum_{\ell=0}^{k}\left(\begin{array}{l}
k \\
\ell
\end{array}\right) \mathcal{W}^{[k+1-\ell]} \mathcal{V}^{[\ell]} \\
& =\sum_{\ell=0}^{k+1}\left(\begin{array}{c}
k \\
\ell-1
\end{array}\right) \mathcal{W}^{[k+1-\ell]} \mathcal{V}^{[\ell]}+\sum_{\ell=0}^{k+1}\left(\begin{array}{l}
k \\
\ell
\end{array}\right) \mathcal{W}^{[k+1-\ell]} \mathcal{V}^{[\ell]} \\
& =\sum_{\ell=0}^{k+1}\left(\begin{array}{c}
k+1 \\
\ell
\end{array}\right) \mathcal{W}^{[k+1-\ell]} \mathcal{V}^{[\ell+1]}
\end{aligned}
$$


which completes the induction step. $\quad \square$

Proof of Lemma 3.

(i) Proof of $(5.9 a)$ :

For $k=0$, identity $(5.9 \mathrm{a})$ follows from

$$
\frac{\mathrm{d}}{\mathrm{d} t} \mathcal{V}^{[0]}=A^{[0]} \mathcal{V}^{[0]}, \quad \text { i.e., } \quad \rho\left(\mathcal{V}^{[0]}\right)=0 .
$$

For the general induction step $k \mapsto k+1$, by the definition of $\mathcal{V}^{[k+1]}$ and due to Lemma 4 (A) we have

$$
\begin{aligned}
\rho\left(\mathcal{V}^{[k+1]}\right) & =\rho\left(\left[\mathcal{V}^{[k]}, \underline{H}\right]\right)+\rho\left(\rho\left(\mathcal{V}^{[k]}\right)\right) \\
& =A^{[1]} \mathcal{V}^{[k]}+\left[\rho\left(\mathcal{V}^{[k]}\right), \underline{H}\right]+\left(\frac{\mathrm{d}}{\mathrm{d} t} \rho\left(\mathcal{V}^{[k]}\right)-A \rho\left(\mathcal{V}^{[k]}\right)\right) .
\end{aligned}
$$

Together with the inductive assumption (5.9a) this yields

$$
\begin{aligned}
\rho\left(\mathcal{V}^{[k+1]}\right) & =A^{[1]} \mathcal{V}^{[k]}+\left(\frac{\mathrm{d}}{\mathrm{d} t} \rho\left(\mathcal{V}^{[k]}\right)-A \rho\left(\mathcal{V}^{[k]}\right)+\left[\rho\left(\mathcal{V}^{[k]}\right), \underline{H}\right]\right) \\
& =A^{[1]} \mathcal{V}^{[k]}+\sum_{\ell=1}^{k}\left(\begin{array}{c}
k \\
\ell
\end{array}\right)\left(\frac{\mathrm{d}}{\mathrm{d} t}\left(A^{[\ell]} \mathcal{V}^{[k-\ell]}\right)-A A^{[\ell]} \mathcal{V}^{[k-\ell]}+\left[A^{[\ell]} \mathcal{V}^{[k-\ell]}, \underline{H}\right]\right),
\end{aligned}
$$

with

$$
\begin{aligned}
& \frac{\mathrm{d}}{\mathrm{d} t}\left(A^{[\ell]} \mathcal{V}^{[k-\ell]}\right)-A A^{[\ell]} \mathcal{V}^{[k-\ell]}+\left[A^{[\ell]} \mathcal{V}^{[k-\ell]}, \underline{H}\right] \\
& =A^{[\ell]} \frac{\mathrm{d}}{\mathrm{d} t} \mathcal{V}^{[k-\ell]}-A A^{[\ell]} \mathcal{V}^{[k-\ell]}+A^{[\ell]}\left[\mathcal{V}^{[k-\ell]}, \underline{H}\right]+\left[A^{[\ell]}, \underline{H}\right] \mathcal{V}^{[k-\ell]} \\
& =A^{[\ell]}\left(A \mathcal{V}^{[k-\ell]}+\rho\left(\mathcal{V}^{[k-\ell]}\right)-A A^{[\ell]} \mathcal{V}^{[k-\ell]}+A^{[\ell]}\left[\mathcal{V}^{[k-\ell]}, \underline{H}\right]+\left[A^{[\ell]}, \underline{H}\right] \mathcal{V}^{[k-\ell]}\right. \\
& =\left[A^{[\ell]}, A\right] \mathcal{V}^{[k-\ell]}+A^{[\ell]} \rho\left(\mathcal{V}^{[k-\ell]}\right)+A^{[\ell]}\left[\mathcal{V}^{[k-\ell]}, \underline{H}\right]+\left[A^{[\ell]}, \underline{H}\right] \mathcal{V}^{[k-\ell]} \\
& =\left[A^{[\ell]}, \underline{H}+A\right] \mathcal{V}^{[k-\ell]}+A^{[\ell]}\left(\left[\mathcal{V}^{[k-\ell]}, \underline{H}\right]+\rho\left(\mathcal{V}^{[k-\ell]}\right)\right) \\
& =A^{[\ell+1]} \mathcal{V}^{[k-\ell]}+A^{[\ell]} \mathcal{V}^{[k+1-\ell]},
\end{aligned}
$$

by the definitions of $A^{[\ell+1]}$ and $\mathcal{V}^{[k+1-\ell]}$. Altogether,

$$
\begin{aligned}
\rho\left(\mathcal{V}^{[k+1]}\right) & =A^{[1]} \mathcal{V}^{[k]}+\sum_{\ell=1}^{k}\left(\begin{array}{l}
k \\
\ell
\end{array}\right)\left(A^{[\ell+1]} \mathcal{V}^{[k-\ell]}+A^{[\ell]} \mathcal{V}^{[k+1-\ell]}\right) \\
& =\sum_{\ell=0}^{k}\left(\begin{array}{c}
k \\
\ell
\end{array}\right) A^{[\ell+1]} \mathcal{V}^{[k-\ell]}+\sum_{\ell=1}^{k}\left(\begin{array}{l}
k \\
\ell
\end{array}\right) A^{[\ell]} \mathcal{V}^{[k+1-\ell]} \\
& =\sum_{\ell=1}^{k+1}\left(\begin{array}{c}
k \\
\ell-1
\end{array}\right) A^{[\ell]} \mathcal{V}^{[k+1-\ell]}+\sum_{\ell=1}^{k+1}\left(\begin{array}{c}
k \\
\ell
\end{array}\right) A^{[\ell]} \mathcal{V}^{[k+1-\ell]} \\
& =\sum_{\ell=1}^{k+1}\left(\begin{array}{c}
k+1 \\
\ell
\end{array}\right) A^{[\ell]} \mathcal{V}^{[k+1-\ell]},
\end{aligned}
$$

which completes the induction step.

(ii) Proof of (5.10a):

For $k=0$, the identity (5.10a) follows from

$$
\frac{\mathrm{d}}{\mathrm{d} t} \mathcal{W}^{[0]}=\mathcal{W}^{[0]} B^{[0]}+C^{[0]} \mathcal{W}^{[0]}, \text { i.e., } \sigma\left(\mathcal{W}^{[0]}\right)=0 .
$$

For the general induction step $k \mapsto k+1$, by the definition of $\mathcal{W}^{[k+1]}$ and due to Lemma 5 (A) we have

$$
\begin{aligned}
\sigma\left(\mathcal{W}^{[k+1]}\right)= & \sigma\left(\left[\mathcal{W}^{[k]}, \underline{H}+D\right]\right)+\sigma\left(\sigma\left(\mathcal{W}^{[k]}\right)\right) \\
= & \mathcal{W}^{[k]} B^{[1]}+C^{[1]} \mathcal{W}^{[k]}+\left[\sigma\left(\mathcal{W}^{[k]}\right), \underline{H}+D\right] \\
& +\left(\frac{\mathrm{d}}{\mathrm{d} t} \sigma\left(\mathcal{W}^{[k]}\right)-\sigma\left(\mathcal{W}^{[k]}\right) B-C \sigma\left(\mathcal{W}^{[k]}\right)\right) .
\end{aligned}
$$


Together with the inductive assumption (5.10a) this yields

$$
\begin{aligned}
\sigma & \left(\mathcal{W}^{[k+1]}\right) \\
= & \left(\mathcal{W}^{[k]} B^{[1]}+C^{[1]} \mathcal{W}^{[k]}\right) \\
& +\left(\frac{\mathrm{d}}{\mathrm{d} t} \sigma\left(\mathcal{W}^{[k]}\right)-\sigma\left(\mathcal{W}^{[k]}\right) B-C \sigma\left(\mathcal{W}^{[k]}\right)+\left[\sigma\left(\mathcal{W}^{[k]}\right), \underline{H}+D\right]\right) \\
= & \left(\mathcal{W}^{[k]} B^{[1]}+C^{[1]} \mathcal{W}^{[k]}\right) \\
& +\sum_{\ell=1}^{k}\left(\begin{array}{c}
k \\
\ell
\end{array}\right)\left(\frac{\mathrm{d}}{\mathrm{d} t}\left(\mathcal{W}^{[k-\ell]} B^{[\ell]}+C^{[\ell]} \mathcal{W}^{[k-\ell]}\right)-\left(\mathcal{W}^{[k-\ell]} B^{[\ell]}+C^{[\ell]} \mathcal{W}^{[k-\ell]}\right) B\right. \\
& \left.\quad-C\left(\mathcal{W}^{[k-\ell]} B^{[\ell]}+C^{[\ell]} \mathcal{W}^{[k-\ell]}\right)+\left[\mathcal{W}^{[k-\ell]} B^{[\ell]}+C^{[\ell]} \mathcal{W}^{[k-\ell]}, \underline{H}+D\right]\right),
\end{aligned}
$$

with

$$
\begin{aligned}
\frac{\mathrm{d}}{\mathrm{d} t} & \left(\mathcal{W}^{[k-\ell]} B^{[\ell]}+C^{[\ell]} \mathcal{W}^{[k-\ell]}\right)-\left(\mathcal{W}^{[k-\ell]} B^{[\ell]}+C^{[\ell]} \mathcal{W}^{[k-\ell]}\right) B \\
& -C\left(\mathcal{W}^{[k-\ell]} B^{[\ell]}+C^{[\ell]} \mathcal{W}^{[k-\ell]}\right)+\left[\mathcal{W}^{[k-\ell]} B^{[\ell]}+C^{[\ell]} \mathcal{W}^{[k-\ell]}, \underline{H}+D\right] \\
= & \frac{\mathrm{d}}{\mathrm{d} t} \mathcal{W}^{[k-\ell]} B^{[\ell]}+C^{[\ell]} \frac{\mathrm{d}}{\mathrm{d} t} \mathcal{W}^{[k-\ell]}-\left(\mathcal{W}^{[k-\ell]} B^{[\ell]}+C^{[\ell]} \mathcal{W}^{[k-\ell]}\right) B \\
& -C\left(\mathcal{W}^{[k-\ell]} B^{[\ell]}+C^{[\ell]} \mathcal{W}^{[k-\ell]}\right)+\left[\mathcal{W}^{[k-\ell]} B^{[\ell]}, \underline{H}+D\right]+\left[C^{[\ell]} \mathcal{W}^{[k-\ell]}, \underline{H}+D\right] \\
= & \left(\mathcal{W}^{[k-\ell]} B+C \mathcal{W}^{[k-\ell]} \sigma\left(\mathcal{W}^{[k-\ell]}\right)\right) B^{[\ell]}+C^{[\ell]}\left(\mathcal{W}^{[k-\ell]} B C \mathcal{W}^{[k-\ell]}+\sigma\left(\mathcal{W}^{[k-\ell]}\right)\right) \\
& -\left(\mathcal{W}^{[k-\ell]} B^{[\ell]}+C^{[\ell]} \mathcal{W}^{[k-\ell]}\right) B-C\left(\mathcal{W}^{[k-\ell]} \ell+C^{[\ell]} \mathcal{W}^{[k-\ell]}\right) \\
& +\mathcal{W}^{[k-\ell]}\left[B^{[\ell]}, \underline{H}+D\right]+\left[\mathcal{W}^{[k-\ell]}, \underline{H}+D\right] B^{[\ell]} \\
& +C^{[\ell]}\left[\mathcal{W}^{[k-\ell]}, \underline{H}+D\right]+\left[C^{[\ell]}, \underline{H}+D\right] \mathcal{W}^{[k-\ell]} \\
= & \left(\mathcal{W}^{[k-\ell]}\left[B, B^{[\ell]}\right]+\mathcal{W}[k-\ell]\left[B^{[\ell]}, \underline{H}+D\right]\right) \\
& +\left(\left[C^{[\ell]}, C\right] \mathcal{W} \mathcal{W}^{[k-\ell]}+\left[C^{[\ell]}, \underline{H}+D\right] \mathcal{W}^{[k-\ell]}\right) \\
& +\left(\left[\mathcal{W}^{[k-\ell]}, \underline{H}+D\right]+\sigma\left(\mathcal{W}^{[k-\ell]}\right)\right) B^{[\ell]} \\
& +C^{[\ell]}\left(\left[\mathcal{W}^{[k-\ell]}, \underline{H}+D\right]+\sigma\left(\mathcal{W}{ }^{[k-\ell]}\right)\right) \\
= & \mathcal{W}^{[k-\ell]}\left[B^{[\ell]}, \underline{H}+A\right]+\left[C^{[\ell]}, \underline{H}+H\right] \mathcal{W}^{[k-\ell]} \\
& +\left(\left[\mathcal{W}^{[k-\ell]}, \underline{H}+D\right]+\sigma\left(\mathcal{W}^{[k-\ell]}\right)\right) B^{[\ell]}+C^{[\ell]}\left(\left[\mathcal{W}^{[k-\ell]}, \underline{H}+D\right]+\sigma\left(\mathcal{W}^{[k-\ell]}\right)\right) \\
= & \left(\mathcal{W}^{[k-\ell]} B^{[\ell+1]}+C^{[\ell+1]} \mathcal{W}^{[k-\ell]}\right)+\left(\mathcal{W}^{[k+1-\ell]} B^{[\ell]}+C^{[\ell]} \mathcal{W}^{[k+1-\ell]}\right),
\end{aligned}
$$

by the definitions of $B^{[\ell+1]}, C^{[\ell+1]}$ and $\mathcal{W}^{[k+1-\ell]}$. Altogether,

$$
\sigma\left(\mathcal{W}^{[k+1]}\right)=\sum_{\ell=1}^{k+1}\left(\begin{array}{c}
k+1 \\
\ell
\end{array}\right)\left(\mathcal{W}^{[k+1-\ell]} B^{[\ell]}+C^{[\ell]} \mathcal{W}^{[k+1-\ell]}\right)
$$

follows in the same same way as in the proof of (5.9a) above, which completes the induction step.

\section{Acknowledgements}

We gratefully acknowledge financial support by the Austrian Science Fund (FWF) under the projects P21620-N13 and P24157-N13. 


\section{References}

1. A. Yochelis, M. Sheintuch, Towards nonlinear selection of reaction-diffusion patterns in presence of advection: a spatial dynamics approach, Phys. Chem. Chem. Phys. 11 (2009) 9210-9223.

2. D. Fournier, J. Sibert, J. Hampton, P. Bills, An advection-diffusion-reaction model for the estimation of fish movement parameters from tagging data, with application to skipjack tuna (katsuwonus pelamis), Canadian Journal of Fisheries and Aquatic Sciences 56 (1999) 925-938.

3. Y. Du, L. Mei, On a nonlocal reaction-diffusion-advection equation modelling phytoplankton dynamics, Nonlinearity 24 (2011) 319-349.

4. W. Bao, Ground states and dynamics of multi-component Bose-Einstein condensates, Multiscale Model. Simul. 2 (2004) 210-236.

5. W. Bao, P. Markowich, C. Schmeiser, R. Weishäupl, On the Gross-Pitaevskii equation with strongly anisotropic confinement: formal asymptotics and numerical experiments, Math. Models Meth. Appl. Sci. 15 (2005) 767-782.

6. W. Bao, H. Wang, An efficient and spectrally accurate numerical method for computing dynamics of rotating Bose-Einstein condensates, J. Comput. Phys. 217 (2006) 612-626.

7. Y. Zhang, W. Bao, H. Li, Dynamics of rotating two-component Bose-Einstein condensates and its efficient computation, Physica D 234 (2007) 49-69.

8. W. Bao, Y. Cai, Mathematical theory and numerical methods for Bose-Einstein condensation, Kinet. Relat. Mod. 6 (2013) 1-135.

9. W. Auzinger, W. Herfort, Local error structures and order conditions in terms of Lie elements for exponential splitting schemes, Opuscula Math. 34 (2014) 243-255.

10. O. Koch, C. Neuhauser, M. Thalhammer, Embedded split-step formulae for the time integration of nonlinear evolution equations, Appl. Numer. Math. 63 (2013), 14-24.

11. W. Auzinger, H. Hofstätter, O. Koch, M. Thalhammer, Defect-based local error estimators for splitting methods, with application to Schrödinger equations, Part III: The nonlinear case, J. Comput. Appl. Math. 273 (2014) 182-204.

12. W. Auzinger, O. Koch, M. Thalhammer, Defect-based local error estimators for splitting methods, with application to Schrödinger equations, Part I: The linear case, J. Comput. Appl. Math. 236 (2012) 2643-2659.

13. W. Auzinger, O. Koch, M. Thalhammer, Defect-based local error estimators for splitting methods, with application to Schrödinger equations, Part II: Higher-order methods for linear problems, J. Comput. Appl. Math. 255 (2013) 384-403.

14. E. Hairer, C. Lubich, G. Wanner, Geometric Numerical Integration, Springer-Verlag, Berlin-Heidelberg-New York, 2002.

15. F. Castella, P. Chartier, S. Descombes, G. Vilmart, Splitting methods with complex times for parabolic equations, BIT Numer. Math. 49 (2009) 487-508.

16. E. Gross, Structure of a quantized vortex in boson systems, Nuovo Cimento 20 (1961) $454-457$.

17. L. Pitaevskii, Vortex lines in an imperfect Bose gas, Soviet Phys. JETP 13 (1961) 451-454

18. A. Doelman, T. Kaper, P. Zegeling, Pattern formation in the one-dimensional Gray-Scott model, Nonlinearity 10 (1997) 523-563.

19. P. Gray, S. Scott, Sustained oscillations and other exotic patterns of behavior in isothermal reactions, J. Phys. Chem. 89 (1985) 22-32. 